\title{
Robust Performance Analysis of Uncertain LTI Systems: Dual LMI Approach and Verifications for Exactness
}

\section{AUTHOR(S):}

Ebihara, Yoshio; Onishi, Yusuke; Hagiwara, Tomomichi

\section{CITATION:}

Ebihara, Yoshio ... [et al]. Robust Performance Analysis of Uncertain LTI Systems: Dual LMI Approach and Verifications for Exactness. IEEE TRANSACTIONS ON AUTOMATIC CONTROL 2009, 54(5): 938-951

\section{ISSUE DATE:}

2009-05

URL:

http://hdl.handle.net/2433/109801

\section{RIGHT:}

(c) 2009 IEEE. Personal use of this material is permitted. However, permission to reprint/republish this material for advertising or promotional purposes or for creating new collective works for resale or redistribution to servers or lists, or to reuse any copyrighted component of this work in other works must be obtained from the IEEE. 


\title{
Robust Performance Analysis of Uncertain LTI Systems: Dual LMI Approach and Verifications for Exactness
}

\author{
Yoshio Ebihara, Member, IEEE, Yusuke Onishi, and Tomomichi Hagiwara, Member, IEEE
}

\begin{abstract}
This paper addresses robust performance analysis problems of linear time-invariant (LTI) systems affected by real parametric uncertainties. These problems, known also as a special class of structured singular value computation problems, are inherently intractable (NP-hard problems). As such intensive research effort has been made to obtain computationally tractable and less conservative analysis conditions, where linear matrix inequality (LMI) plays an important. Nevertheless, since LMI-based conditions are expected to be conservative in general, it is often the case that we cannot conclude anything if the LMI at hand turns out to be infeasible. This motivates us to consider the dual of the LMI and examine the structure of the dual solution. By pursuing this direction, in this paper, we provide rank conditions on the dual solution matrix under which we can conclude that the underlying robust performance is never attained. In particular, a set of uncertain parameters that violates the specified performance can be computed. These results come from block-moment matrix structure of the dual variable, which is consistent with the recent results on polynomial optimization. This particular structure enables us to make good use of simultaneous diagonalizability property of commuting diagonalizable matrices so that the sound rank conditions for the exactness verification can be obtained.
\end{abstract}

Index Terms-Block-Moment matrix structure, dual linear matrix inequalities (LMIs), exactness verification, robust performance analysis.

\section{INTRODUCTION}

$\mathbf{R}$ OBUSTNESS analysis of linear time-invariant (LTI) systems affected by real parametric uncertainties has been a challenging topic in the community of control theory. The general framework of $\mu$ theory [25] has been developed to deal with this problem, leading to sophisticated techniques based on scalings [23]. These studies clarified that those computationally efficient scaling-based approaches are conservative, except for some specific cases where the number of the uncertain parameters and their structures satisfy certain conditions. Indeed, it is now well-recognized that most of practical robustness analysis problems are NP-hard problems and hence computationally intractable.

Manuscript received March 10, 2007; revised February 13, 2008. Current version published May 13, 2009. This work was supported in part by the Ministry of Education, Culture, Sports, Science and Technology of Japan under Grant-in-Aid for Young Scientists (B), 18760319. Recommended by Guest Editors G. Chesi and D. Henrion.

Y. Ebihara and T. Hagiwara are with the Department of Electrical Engineering, Kyoto University, Kyoto 615-8510, Japan (e-mail: ebihara@kuee.kyoto-u.ac.jp; hagiwara@kuee.kyoto-u.ac.jp).

Y. Onishi is with the Kansai Telecasting Corporation, Osaka 530, Japan (e-mail: onishi@jaguar.kuee.kyoto-u.ac.jp).

Digital Object Identifier 10.1109/TAC.2009.2017086
As the effectiveness of the linear matrix inequality (LMI) has been revealed for the analysis and synthesis of uncertainty-free systems [4], its robust counterpart, so called robust LMI [5], [11], [29]-[31], emerges as a powerful tool to deal with robustness issues. Since robustness analysis and synthesis problems enforce us to deal with transfer functions or state space equations affected by uncertainties, a natural problem formulation leads to robust LMIs, where the coefficient matrices depend on the uncertain parameters. In particular, intensive research effort has been made for robust LMIs with coefficient matrices depending rationally upon the uncertain parameters [18], [20], [28], which certainly capture whole variety of robustness analysis and synthesis problems.

When dealing with robust LMIs describing infinitely many constraints, the key issue is how to reduce them into numerically verifiable finitely many LMIs. This step to remove the semi-infinite constraints is called relaxation in the literature [15], [16], [29]-[32]. In the simplest case where the robust LMI involves only a single parameter, the celebrated Kalman-Yakubovich-Popov Lemma [27] enables us to derive a desired LMI in an exact fashion. In other cases, however, it is far from achievable to obtain LMIs with a priori certificate for the exactness [29]-[32]. This poses essential limitations on the robustness analysis of linear systems, since linear systems inherently involve a frequency parameter. It follows that we cannot expect exact LMIs even when the underlying robustness is against only a single uncertain parameter (unless it has the full complex structure as in the standard $H_{\infty}$ norm computation [23]). Thus, the LMI resulting from relaxation is conservative in general. Therefore it is often the case that we cannot conclude anything directly if the LMI at hand turns out to be infeasible via numerical computation.

To cope with this difficult situation, a novel approach was proposed by Scherer [29]-[31]. The key idea is to take the "dual" of the "primal" LMI resulting from relaxation and examine the dual solution, which does exist if the primal LMI is infeasible. The result in [29], [30] is such that if the computed dual solution satisfies a certain condition, then we can conclude the existence of the worst case perturbation that violates the underlying robust LMI. Recall that, in nonlinear programming, it is surely a fundamental strategy to investigate the property of computed solutions and examine their optimality [3]. Scherer's works realize this in an elegant fashion within the framework of robust LMIs.

In this paper, we pursue the direction related to but yet distinct from [29]-[31], inspired by the recent results on polynomial optimization [15], [22], [26] and positivity analysis of polynomial 
matrices [8]. Namely, we certainly follow the basic strategy to obtain numerically verifiable LMI via relaxation and take its dual for the exactness verification. What makes the present approach novel is summarized as follows:

1) Exactness Verification for Infinite-Dimensional Robust LMI Problems: Robustness analysis problems of LTI systems affected by parametric uncertainties are naturally formulated as robust LMI problems involving parameter-dependent multipliers [7] (those multipliers are often understood in relation with the parameter-dependent and quadratic in the state Lyapunov functions). Namely, our robust LMIs are infinite-dimensional from the outset, since we have to seek for the desired multiplier over an infinite-dimensional function space. One of the standard idea to get around this difficulty is to obtain a finite-dimensional robust LMI by restricting the search of the multiplier to some finite-dimensional subspace. Due to this restriction, however, the infeasibility of the resulting finite-dimensional robust LMI does not necessarily imply the infeasibility of the original one. Thus, we surely need another effort, and in this paper we provide a novel methodology for the exactness verification dedicated to the infinite-dimensional robust LMI problems arising in robustness analysis of uncertain LTI systems.

2) Modification of the $(D, G)$ Scaling in Multiple Uncertain Parameter Cases: This modification is technically trivial but yet of great importance in the present dual LMI approach. Namely, by deriving a primal LMI from a robust LMI via $(D, G)$ scaling with proper modifications, we will show that the corresponding dual variable has a block-matrix structure that is consistent with the moment matrix in [16], [22]. In particular, this block-moment matrix structure enables us to derive a sound rank condition for the exactness verification. Our result ensures that, if the computed dual variable satisfies the suggested rank condition, then the underlying robust performance is never attained. More specifically, we can readily compute a set of uncertain parameters that violates the specified performance. The key idea to derive these results comes from simultaneous diagonalizability property of commuting diagonalizable matrices [17]. The block-moment matrix structure plays an essential role to make good use of this property.

As stated, the present results could be regarded as partial extension of those in [22] to polynomial matrix cases. Specific connections to these recent results on polynomial optimization via sum-of-squares (SOS) relaxation [15], [22], [26] as well as their matrix counterparts [16], [29]-[32] are concretely discussed in the latter part of the paper. Throughout the paper, particular emphasis is laid upon practical usefulness, and we will illustrate via extensive numerical experiments that the suggested rank conditions work effectively to detect the worst case perturbations and conclude the exactness.

We use the following notations in this paper. For a matrix $A \in \mathbf{R}^{n \times n}$, $\operatorname{He}\{A\}$ is a shorthand notation for $A+A^{T}$, and $\lambda(A)$ denotes the set of the eigenvalues of $A$. For a matrix $B \in$ $\mathbf{R}^{n \times m}, B^{\dagger}$ stands for its Moore-Penrose generalized inverse [17], and $B^{\perp} \in \mathbf{R}^{(n-r) \times n}$ is a matrix such that $B^{\perp} B=0$ and $B^{\perp}\left(B^{\perp}\right)^{T}>0$ where $r:=\operatorname{rank}(B)$. The symbols $\mathbf{S}_{n}, \mathbf{P}_{n}$ and $\mathbf{S k}_{n}$ denote the set of real symmetric, real positive-semidefinite and real skew-symmetric matrices of the size $n$, respectively. For $A \in \mathbf{S}_{n}$, we denote by $\lambda_{k}(A)(k=1, \ldots, n)$ the $k$-th eigenvalue of $A$. Finally, for a matrix $A \in \mathbf{R}^{\left(n_{1}+n_{2}\right) \times\left(m_{1}+m_{2}\right)}$ with partition

$$
A=\left[\begin{array}{ll}
A_{11} & A_{12} \\
A_{21} & A_{22}
\end{array}\right], \quad A_{11} \in \mathbf{R}^{n_{1} \times m_{1}}, \quad A_{22} \in \mathbf{R}^{n_{2} \times m_{2}}
$$

we define $\lceil A\rfloor^{n_{1}}:=\left[\begin{array}{ll}A_{11} & A_{12}\end{array}\right]$ and $\lceil A\rfloor_{n_{2}}:=\left[\begin{array}{ll}A_{21} & A_{22}\end{array}\right]$. In particular, if $A$ is square and $n_{i}=m_{i}(i=1,2)$, we define $\langle A\rangle^{n_{1}}:=A_{11}$ and $\langle A\rangle_{n_{2}}:=A_{22}$.

\section{PROBlem Formulation AND FundAMENTAl RESUlts}

\section{A. Problem Formulation}

Throughout the paper, we will consider the problem described below:

Problem: For given $\Theta \subset \mathbf{R}^{L}$ and a rational function $M(\theta)$ : $\mathbf{R}^{L} \rightarrow \mathbf{R}^{n \times n}$, determine whether $\{\lambda(M(\theta)) \cap j \mathbf{R}\}=\phi$ holds for all $\theta \in \Theta$.

Obviously, this problem arises in robust stability analysis problems of continuous-time LTI systems affected by the uncertain parameter $\theta \in \Theta$. In fact, the scope of this problem formulation is quite broad and includes robust dissipation performance analysis, which can be seen using the idea of Hamiltonian eigenvalue tests [12], [35]. We note here that the above problem tacitly involves "frequency parameter $s$," since the condition $\{\lambda(M(\theta)) \cap j \mathbf{R}\}=\phi$ can be restated equivalently as $\operatorname{det}(s I-M(\theta)) \neq 0(\forall s \in j \mathbf{R})$.

\section{B. Fundamental Results}

Our primary concern is to derive numerically verifiable conditions to provide yes/no answers to the aforementioned problem. To suggest the direction of our approach and explicate the underlying idea, in this subsection, let us consider a fairly simplified but still insightful problem described in the following:

Problem 1: For given $M_{0}, M_{1} \in \mathbf{R}^{n \times n}$ and $\delta>0$, determine whether $\left\{\lambda\left(M_{0}+\theta M_{1}\right) \cap j \mathbf{R}\right\}=\phi$ holds for all $\theta \in \Theta_{\delta}:=[-\delta, \delta]$.

In the case of robust stability analysis, the matrix $M_{0}$ is naturally assumed to be Hurwitz stable. Hence, via continuity arguments of the eigenvalues of $M_{0}+\theta M_{1}$, Problem 1 can be reduced, with somewhat tricky transformations, into a numerically tractable generalized eigenvalue computation problem [2]. However, in robust performance analysis cases (ex., Hamiltonian eigenvalue tests), those computation methods [2] specialized to robust stability analysis cannot be applied. Thus, in contrast with its simple description, Problem 1 is still intractable.

To get around this difficulty, one of the promising way is to recast Problem 1 into a robust LMI problem [5], [11], [29]-[31]. Namely, it can be shown that $\left\{\lambda\left(M_{0}+\theta M_{1}\right) \cap j \mathbf{R}\right\}=\phi(\forall \theta \in$ $\Theta_{\delta}$ ) holds if and only if there exists a matrix $P(\theta): \mathbf{R} \rightarrow \mathbf{S}_{n}$ such that

$$
\operatorname{He}\left\{P(\theta)\left(M_{0}+\theta M_{1}\right)\right\} \prec 0 \quad \forall \theta \in \Theta_{\delta} .
$$

The matrix $P(\theta)$ is often called a parameter-dependent multiplier [7]. In this way, we can reformulate Problem 1 into an infinite-dimensional robust LMI problem.

It is known that, since $\Theta_{\delta}$ is compact, $P(\theta)$ in (1) can be taken as a polynomial [6]. In particular, once we have restricted 
our attention to the finite-degree polynomial approximation $P_{N}(\theta):=\sum_{i=0}^{N} \theta^{i} P_{i}$, the corresponding problem to (1) can be reduced to a finite-dimensional LMI problem via $(D, G)$ scaling [21], [23]. However, the degree $N$ that ensures exact analysis is not known in general, even though specific results have been obtained in robust stability analysis cases [14], [34]. In view of this fact, all we can do amounts to constructing numerically verifiable finite-dimensional LMI problems that are conservative in general (i.e., there is no a priori certificate for the exactness). Our approach is such that, when an LMI at hand turns out to be infeasible and we cannot conclude anything directly, we try to conclude "no" by detecting the worst case perturbation that violates the underlying performance criterion.

To explicate our basic approach, let us consider $N$-th degree polynomial approximation $P(\theta)=P_{N}(\theta)$ in (1) where $N$ is an odd number. Then, via $(D, G)$ scaling, we can reduce (1) into a finite-dimensional LMI problem described below:

Primal LMI Problem: Find $P_{i} \in \mathbf{S}_{n}(i=0, \ldots, N), D \in$ $\mathbf{P}_{(N+1) n / 2}$ and $G \in \mathbf{S k}_{(N+1) n / 2}$ such that

$$
\begin{aligned}
\left.\operatorname{He}\left\{\begin{array}{cccc}
P_{0} & 0 & \cdots & 0 \\
P_{1} & P_{2} & \ddots & \vdots \\
0 & P_{3} & \ddots & 0 \\
\vdots & \ddots & \ddots & P_{N-1} \\
0 & \cdots & 0 & P_{N}
\end{array}\right]\left[\begin{array}{ccccc}
M_{0} & M_{1} & 0 & \cdots & 0 \\
0 & M_{0} & M_{1} & \ddots & \vdots \\
\vdots & \ddots & \ddots & \ddots & 0 \\
0 & \cdots & 0 & M_{0} & M_{1}
\end{array}\right]\right\} \\
+J_{n, N}\left[\begin{array}{cc}
\delta^{2} D & G \\
G^{T} & -D
\end{array}\right] J_{n, N}^{T} \prec 0
\end{aligned}
$$

where $J_{n, N}:=\left[\begin{array}{ll}\hat{J}_{n, N} & \check{J}_{n, N}\end{array}\right] \in \mathbf{R}^{(N+3) n / 2 \times(N+1) n}$ with $\hat{J}_{n, N}:=\left[\begin{array}{ll}I_{(N+1) n / 2} & 0_{(N+1) n / 2, n}\end{array}\right]^{T}$ and $\check{J}_{n, N}:=$ $\left[0_{(N+1) n / 2, n} I_{(N+1) n / 2}\right]^{T}$. Our approach relies on its dual, which readily follows from the convex duality theory [1].

Dual LMI Problem: Find $\mathcal{H} \in \mathbf{S}_{(N+3) n / 2} \backslash\{0\}$ such that

$$
\mathcal{H}=\left[\begin{array}{cccc}
\mathcal{H}_{0} & \mathcal{H}_{1} & \ldots & \mathcal{H}_{(N+1) / 2} \\
\mathcal{H}_{1} & \therefore & \therefore & \vdots \\
\vdots & \therefore & \therefore & \mathcal{H}_{N} \\
\mathcal{H}_{(N+1) / 2} & \cdots & \mathcal{H}_{N} & \mathcal{H}_{N+1}
\end{array}\right] \succeq 0
$$

$$
\delta^{2} \overline{\mathcal{H}}-\underline{\mathcal{H}} \succeq 0, \quad \overline{\mathcal{H}}:=\langle\mathcal{H}\rangle^{(N+1) n / 2}, \quad \underline{\mathcal{H}}:=\langle\mathcal{H}\rangle_{(N+1) n / 2}
$$$$
\operatorname{He}\left\{M_{0} \mathcal{H}_{j}+M_{1} \mathcal{H}_{j+1}\right\}=0(j=0, \ldots N) \text {. }
$$

As shown in (3), the dual problem involves LMIs and linear matrix equalities (LMEs) with respect to the dual variable $\mathcal{H}$, which has the block-Hankel matrix structure. Since the primal LMI (2) is strict, exactly one of the primal and dual LMI is feasible [1], [31]. This relation is referred to as strict alternative [1], and tacitly used in the sequel.

Having described the dual problem, we are now ready to state the first result in this paper. The following result forms an important basis of our study and motivates us to explore its further extensions as described in Sections III and IV.

Theorem 1:

i) Suppose (3) is infeasible. Then, $\left\{\lambda\left(M_{0}+\theta M_{1}\right) \cap j \mathbf{R}\right\}=$ $\phi$ holds for all $\theta \in \Theta_{\delta}$. ii) Suppose (3) is feasible and has a solution $\mathcal{H}$. Then, if

$$
\operatorname{rank}(\overline{\mathcal{H}})=\operatorname{rank}(\mathcal{H})
$$

there exists $\theta_{\mathrm{w}} \in \Theta_{\delta}$ such that $\left\{\lambda\left(M_{0}+\theta_{\mathrm{w}} M_{1}\right) \cap j \mathbf{R}\right\} \neq$ $\phi$. More precisely, if we denote the full-rank factorization of $\mathcal{H}$ by $\mathcal{H}=H H^{T}\left(H \in \mathbf{R}^{(N+3) n / 2 \times m}\right)$ and define $\bar{H}:=\lceil H\rfloor^{(N+1) n / 2}, \underline{H}:=\lceil H\rfloor_{(N+1) n / 2}$ and $\Omega:=\bar{H}^{\dagger} \underline{H}$, then $\Omega \in \mathbf{S}_{m}$ and $\lambda(\Omega) \subset \Theta_{\delta}$. In addition, we have $\left\{\lambda\left(M_{0}+\lambda_{k}(\Omega) M_{1}\right) \cap j \mathbf{R}\right\} \neq \phi$ for all $k=1, \ldots, m$.

iii) If there exists $\theta_{\mathrm{w}} \in \Theta_{\delta}$, then there exists $\mathcal{H} \in$ $\mathbf{S}_{(N+3) n / 2} \backslash\{0\}$ such that (3) and (4) hold.

The result i) readily follows from strict alternative. It should be also elementary to see that iii) holds. Indeed, if $\left(M_{0}+\theta_{\mathrm{w}} M_{1}\right) h=0$ holds for $\theta_{\mathrm{w}} \in \Theta_{\delta}$ and $h \in \mathbf{R}^{n} \backslash\{0\}$, the conditions (3) and (4) are satisfied with $\mathcal{H}=H H^{T}$ where $H=\left[\begin{array}{llll}h^{T} & \theta_{\mathrm{w}} h^{T} & \ldots & \theta_{\mathrm{w}}^{(N+1) / 2} h^{T}\end{array}\right]^{T}$. Similarly, if $\left(M_{0}+\theta_{\mathrm{w}} M_{1}\right) h=j \omega h$ holds for $\theta_{\mathrm{w}} \in \Theta_{\delta}, \omega \in \mathbf{R} \backslash\{0\}$ and $h \in \mathbf{C}^{n} \backslash\{0\}$, the conditions are satisfied with $\mathcal{H}=H H^{T}$ where

$$
H=\left[\begin{array}{cc}
\operatorname{Re}(h) & \operatorname{Im}(h) \\
\operatorname{Re}(h) \theta_{\mathrm{w}} & \operatorname{Im}(h) \theta_{\mathrm{w}} \\
\vdots & \vdots \\
\operatorname{Re}(h) \theta_{\mathrm{w}}^{(N+1) / 2} & \operatorname{Im}(h) \theta_{\mathrm{w}}^{(N+1) / 2}
\end{array}\right]
$$

Note that the above construction of $\mathcal{H}$ provides an alternative proof for i).

The importance of the theorem lies in the result ii), where we have given a rank condition on the dual variable $\mathcal{H}$ under which the existence of the worst case perturbation is ensured. In particular, the worst case perturbation can be obtained by simply constructing $\Omega$ from $\mathcal{H}$ and computing its eigenvalues. Thus, in contrast with the standard approach based on the primal LMI, we can ensure the existence of the worst case perturbation. From extensive numerical experiments, we can confirm that the suggested rank condition surely works effectively to detect the worst case perturbation and conclude the exactness of the analysis results. See Section II-C for illustration.

To prove the result ii), and to extend Theorem 1 to rationaldependence and/or multiple uncertain parameter cases in Sections III and IV, the notion of simultaneous diagonalizability of a commuting diagonalizable matrix family plays a crucial role. The definition and related results are reviewed.

Definition 1: [17] A family $\mathbf{M} \subset \mathbf{C}^{n \times n}$ of matrices is an arbitrary (finite or infinite) set of matrices, and a commuting family is one in which each pair in the set commutes under multiplication. A simultaneously diagonalizable family is one for which there is a single nonsingular matrix $U \in \mathbf{C}^{n \times n}$ such that $U^{-1} A U$ is diagonal for every $A \in \mathbf{M}$.

Lemma 1: [17] Let $\mathbf{M}$ be a family of diagonalizable matrices. Then $\mathbf{M}$ is a commuting family if and only if it is a simultaneously diagonalizable family.

In addition to Lemma 1, the next two lemmas play important roles. These results are closely related to and strongly inspired from that of [19], [23], [27]. The proofs are given in Appendix section.

Lemma 2: For given $F, G \in \mathbf{R}^{n \times m}$, the following two statements are equivalent: 
i) $G G^{T} \preceq F F^{T}$ and $F G^{T}=G F^{T}$.

ii) There exists $\Omega \in \mathbf{S}_{m}$ with $\|\Omega\| \leq 1$ such that $G=F \Omega$.

Lemma 3: For given $F, G \in \mathbf{R}^{n \times m}$ with $F$ being full-column rank, the following two statements are equivalent:

i) $F G^{T}+G F^{T}=0$.

ii) There exists $\Gamma \in \mathbf{S k}_{m}$ such that $G=F \Gamma$.

We are now ready to prove ii) in Theorem 1 . We note that the proof relies on the particular block-Hankel matrix structure of $\mathcal{H}$ in (3).

Proof of ii) in Theorem 1: First of all, we note from the block-Hankel matrix structure of $\mathcal{H}$ in (3) that the matrices $\bar{H}$ and $\underline{H}$ satisfy $\underline{H} \underline{H}^{T} \preceq \delta^{2} \bar{H} \bar{H}^{T}$ and $\bar{H} \underline{H}^{T}=\underline{H} \bar{H}^{T}$. Hence, from Lemma 2, there exists $\Omega \in \mathbf{S}_{m}$ with $\|\Omega\| \leq \delta$ such that $\underline{H}=\bar{H} \Omega$. This implies that the matrix $H \in \mathbf{R}^{(N+3) n / 2 \times m}$ can be written in the form of $H=\left[\begin{array}{llll}H_{0}^{T} & \Omega H_{0}^{T} & \ldots & \Omega^{(N+1) / 2} H_{0}^{T}\end{array}\right]^{T}\left(H_{0} \in \mathbf{R}^{n \times m}\right)$. In addition, careful inspection on the last $N+1$ equalities in (3) yields

$$
\begin{gathered}
\text { He }\left\{\left[\begin{array}{c}
M_{0} H_{0}+M_{1} H_{0} \Omega \\
\vdots \\
\left(M_{0} H_{0}+M_{1} H_{0} \Omega\right) \Omega^{(N-1) / 2}
\end{array}\right] \Omega^{k}\right. \\
\left.\times\left[\begin{array}{c}
H_{0} \\
\vdots \\
H_{0} \Omega^{(N-1) / 2}
\end{array}\right]\right\}=0(k=0,1) .
\end{gathered}
$$

Since we see from (4) that $\bar{H}=\left[\begin{array}{lll}H_{0}^{T} & \ldots & \Omega^{(N-1) / 2} H_{0}^{T}\end{array}\right]^{T}$ is of full-column rank, the above two equalities and Lemma 3 ensure the existence of $\Gamma, \Gamma_{1} \in \mathbf{S k}_{m}$ such that

$$
\begin{gathered}
{\left[\begin{array}{c}
M_{0} H_{0}+M_{1} H_{0} \Omega \\
\vdots \\
\left(M_{0} H_{0}+M_{1} H_{0} \Omega\right) \Omega^{(N-1) / 2}
\end{array}\right]=\bar{H} \Gamma} \\
{\left[\begin{array}{c}
M_{0} H_{0}+M_{1} H_{0} \Omega \\
\vdots \\
\left(M_{0} H_{0}+M_{1} H_{0} \Omega\right) \Omega^{(N-1) / 2}
\end{array}\right] \Omega=\bar{H} \Gamma_{1} .}
\end{gathered}
$$

Thus, $\bar{H} \Gamma \Omega=\bar{H} \Gamma_{1}$ is satisfied. Again, since $\bar{H}$ is full-column rank, we have $\Gamma \Omega=\Gamma_{1}$, which implies $\Gamma \Omega=\Omega \Gamma$. Namely, the family $\{\Gamma, \Omega\}$ is a commuting family. In addition, since $\Gamma \in \mathbf{S k}_{m}$ and $\Omega \in \mathbf{S}_{m}$ are both normal matrices and hence unitary diagonalizable, Lemma 1 ensures that they share common eigenvectors $u_{k} \in \mathbf{C}^{m}(k=1, \ldots, m)$ as follows:

$$
\begin{aligned}
& \Gamma u_{k}=\lambda_{\Gamma, k} u_{k} \quad \lambda_{\Gamma, k} \in j \mathbf{R} \\
& \Omega u_{k}=\lambda_{k}(\Omega) u_{k} \quad \lambda_{k}(\Omega) \in \Theta_{\delta} .
\end{aligned}
$$

To complete the proof, let us multiply the common eigenvector $u_{k}$ to the equality of the first row in (6) from right, which yields

$$
\begin{aligned}
\left(M_{0}+\lambda_{k}(\Omega) M_{1}\right) x_{k} & =\lambda_{\Gamma, k} x_{k} \\
x_{k} & :=H_{0} u_{k} \in \mathbf{C}^{n}(k=1, \ldots, m) .
\end{aligned}
$$

It should be noted that $x_{k} \neq 0(k=1, \ldots, m)$ since $\bar{H}=\left[\begin{array}{lll}H_{0}^{T} & \ldots & \Omega^{(N-1) / 2} H_{0}^{T}\end{array}\right]^{T}$ is full-column rank. The (8) clearly indicates that the assertions in ii) hold.
In the preceding studies for the exactness verifications of LMI relaxations, so called rank-one exactness principle is derived (see related discussions in [13], [30] and references therein). In our context, this requires $\operatorname{rank}(\mathcal{H})=1$. It is obvious that if $\operatorname{rank}(\mathcal{H})=1$, then $\operatorname{rank}(\overline{\mathcal{H}})=\operatorname{rank}(\mathcal{H})=1$ and thus $(4)$ is automatically satisfied. Namely, the suggested rank-condition is more general than the rank-one principle, and this generalization is quite important to detect the worst case perturbation as shown in Section II-C.

It is meaningful to examine the mutual connections among the solutions of (3) for different degree $N$. In particular, it is preferable if the required rank-condition becomes more likely to be satisfied by increasing the degree $N$. This is indeed the case, and for its formal statement, we define the following map for given odd number $N$ :

$$
\begin{gathered}
\mathcal{H}_{\mathrm{H}}^{N}: \mathbf{R}^{n \times \hat{m}} \times \mathbf{S}_{\hat{m}} \rightarrow \mathbf{S}_{(N+3) n / 2} \\
\mathcal{H}_{\mathrm{H}}^{N}\left(H_{0}, \Omega\right)=\left[\begin{array}{c}
H_{0} \\
\vdots \\
H_{0} \Omega^{(N+1) / 2}
\end{array}\right]\left[\begin{array}{c}
H_{0} \\
\vdots \\
H_{0} \Omega^{(N+1) / 2}
\end{array}\right]^{T} .
\end{gathered}
$$

Then, from the proof of Theorem 1, we see that the next result holds.

Corollary 1: For every $\mathcal{H}$ satisfying (3), there exist $H_{0} \in$ $\mathbf{R}^{n \times \hat{m}}$ and $\Omega \in \mathbf{S}_{\hat{m}}$ such that $\mathcal{H}=\mathcal{H}_{\mathrm{H}}^{N}\left(H_{0}, \Omega\right)$.

With this result in mind, we state the next theorem. The proof is given in Appendix section.

Theorem 2: For given positive odd numbers $N_{1}, N_{2}$ with $N_{1} \leq N_{2}, H_{0} \in \mathbf{R}^{n \times \hat{m}}$ and $\Omega \in \mathbf{S}_{\hat{m}}$, if $\mathcal{H}_{\mathrm{H}}^{N_{2}}\left(H_{0}, \Omega\right)$ satisfies (3) for $N=N_{2}$, then $\mathcal{H}_{\mathrm{H}}^{N_{1}}\left(H_{0}, \Omega\right)$ satisfies (3) for $N=N_{1}$. Conversely, if $\mathcal{H}_{\mathrm{H}}^{N_{1}}\left(H_{0}, \Omega\right)$ satisfies (3) and (4) for $N=N_{1}$, then $\mathcal{H}_{\mathrm{H}}^{N_{2}}\left(H_{0}, \Omega\right)$ satisfies (3) and (4) for $N=N_{2}$.

This theorem clearly shows that the higher-degree polynomial multiplier appropriately restricts the dual solution while loosens the rank condition for the exactness verification so that, if the dual solution exists, the worst case perturbation detection can be highly expected. We illustrate this through numerical experiments.

Before proceeding to numerical experiments, we briefly discuss how the rank condition varies when we employ a polynomial multiplier of even degree $N_{\mathrm{e}}$. In this case, the corresponding dual problem becomes (3) for $N=N_{\mathrm{e}}+1$ with the last $N_{\mathrm{e}}+2$ equalities replaced by $N_{\mathrm{e}}+1$ equalities $\operatorname{He}\left\{M_{0} \mathcal{H}_{j}+\right.$ $\left.M_{1} \mathcal{H}_{j+1}\right\}=0\left(j=0, \ldots, N_{\mathrm{e}}\right)$. Unfortunately, this does not allow us to ensure (5) for $k=1$ and hence the rank condition for the exactness verification degenerates into

$$
\operatorname{rank}\left(\langle\mathcal{H}\rangle^{N_{\mathrm{e}} n / 2}\right)=\operatorname{rank}(\mathcal{H}) .
$$

It follows that if $\mathcal{H} \in \mathbf{S}_{\left(N_{\mathrm{e}}+4\right) n / 2}$ is a solution of the dual problem for the $N_{\mathrm{e}}$-th degree multiplier and satisfies the rank condition (9), then the matrix $\langle\mathcal{H}\rangle^{\left(N_{\mathrm{e}}+2\right) n / 2}$ also satisfies (3) and (4) for $N=N_{\mathrm{e}}-1$. In other phrase, even if we increase the degree from $N_{\mathrm{e}}-1$ to $N_{\mathrm{e}}$, we cannot theoretically go beyond the exactness verification test in the case of $\left(N_{\mathrm{e}}-1\right)$-th degree multiplier. This rather unexpected result stems from the $(D, G)$ scaling where the size of the dual LMI grows when we increase the degree from $N_{\mathrm{e}}-1$ to $N_{\mathrm{e}}$ while remains the same when we increase from $N_{\mathrm{e}}$ to $N_{\mathrm{e}}+1$. For this technical reason, we stated the results only for the case of odd degree polynomial 
multipliers. Note however that, in practical numerical computation, it is often observed that the matrix $\mathcal{H}$ obtained by solving the dual problem for the $N_{\mathrm{e}}$-th degree surely satisfies the desired rank condition, even for those problems where the rank condition fails when we solve the dual problem for the $\left(N_{\mathrm{e}}-1\right)$-th degree. See the numerical experiments discussed in II-C2 for illustration.

\section{Numerical Experiments}

1) Randomly Generated Matrix Pairs: To examine how the suggested rank-condition works effectively to detect the worst case perturbation in practice, we first solve (3) for randomly generated 100 matrix pairs $M_{0}, M_{1} \in \mathbf{R}^{5 \times 5}$ with $N=1$ and $\delta=1$. We generate $M_{0}$ and $M_{1}$ by using MATLAB command rand so that all of their elements lie on $[-1,1]^{1}$. It turns out that in 90 cases out of 100 cases, (3) is feasible and among them, in 57 cases, the rank-condition (4) is satisfied. The average CPU time to determine feasibility/infeasibility was 0.24 [sec].

For example, for the matrices

$$
\begin{aligned}
M_{0} & =\left[\begin{array}{rrrrr}
-0.4 & 0.7 & -0.8 & 0.2 & -0.4 \\
-0.6 & 0.1 & 0.3 & 0.0 & 0.4 \\
0.2 & -0.2 & 0.4 & -0.7 & -0.6 \\
-0.3 & 0.1 & 0.2 & 0.3 & 0.0 \\
0.0 & 0.2 & 0.0 & 0.8 & 0.8
\end{array}\right] \\
M_{1} & =\left[\begin{array}{rrrrr}
-0.1 & 0.1 & 0.5 & -0.3 & 0.4 \\
0.5 & -0.1 & 0.2 & -0.2 & 0.6 \\
-0.3 & 0.4 & -0.1 & -0.6 & 0.2 \\
-0.1 & -0.3 & -0.5 & -0.4 & 0.1 \\
0.1 & 0.9 & 0.2 & 0.0 & 0.5
\end{array}\right]
\end{aligned}
$$

the matrix $\mathcal{H}$ resulting from (3) is numerically verified to satisfy $\operatorname{rank}\left(\mathcal{H}_{0}\right)=\operatorname{rank}(\mathcal{H})=4$ (via singular value decomposition). The matrix $\Omega=H_{0}^{\dagger} H_{1}$ is

$$
\Omega=\left[\begin{array}{crrr}
0.5543 & 0.0654 & -0.2867 & 0.1438 \\
0.0654 & -0.1943 & -0.0423 & -0.0227 \\
-0.2867 & -0.0423 & -0.1175 & -0.0328 \\
0.1438 & -0.0227 & -0.0328 & -0.1695
\end{array}\right]
$$

where $\lambda(\Omega)=\{0.6908,-0.1529,-0.2324,-0.2324\}$. We can confirm that $\left\{\lambda\left(M_{0}+\lambda_{k}(\Omega) M_{1}\right) \cap j \mathbf{R}\right\} \neq \phi$ surely holds for all $k=1, \ldots, 4$.

We should say that the above results are not satisfactory since we cannot conclude anything in the rest 33 cases. To make the rank-condition more likely to be satisfied, we next apply the following well-known heuristic [15]:

$$
\text { minimize } \operatorname{trace}(\mathcal{H}) \text { subject to }(3) \text { and } \operatorname{trace}\left(\mathcal{H}_{0}\right)=1 \text {. }
$$

With this trace minimization, the rank of $\mathcal{H}$ tends to be reduced and thus we can expect that the suggested rank-condition becomes more likely to be satisfied. Indeed, by solving (11) for the same 100 matrix pairs with $N=1$, it turned out that in 85 cases out of the feasible 90 cases the rank condition (4) is satisfied. The average CPU time was 0.23 [sec]. Moreover, if we let $N=3$, the rank condition (4) is satisfied for all 90 feasible cases. Computational burden is still moderate and the average CPU time was 0.32 [sec].

\footnotetext{
${ }^{1}$ In this paper, all LMI-related computation was carried out with SeDuMi [33] and MATLAB R2006a, on PC with CPU Pentium IV 3.6 GHz.
}

Next, let us consider the following problem:

$$
\begin{aligned}
\text { compute } \delta_{\max } & :=\max \delta \text { such that } \\
\left\{\lambda\left(M_{0}+\theta M_{1}\right) \cap j \mathbf{R}\right\} & =\phi \quad \forall \theta \in(-\delta, \delta) .
\end{aligned}
$$

In robust stability/performance analysis, we are typically required to compute stability/performance margins, and the problem setting (12) conforms to this requirement. To solve (12) for the same 100 matrix pairs as above, we carried out a bisection search over $\delta$ in (11) with $N=1$. At the minimal value of $\delta$ for which the existence of $\mathcal{H}$ is ensured, we examined whether the rank condition (4) is satisfied. Then, in 72 cases $\operatorname{rank}\left(\mathcal{H}_{0}\right)=\operatorname{rank}(\mathcal{H})=1$ holds while in the rest 28 cases, $\operatorname{rank}\left(\mathcal{H}_{0}\right)=\operatorname{rank}(\mathcal{H})=2$. Namely, in every case, we can ensure the exactness of the computed margins even with $N=1$. In particular, in the former case, one of the eigenvalues of $M_{0}+\theta M_{1}$ becomes zero at $\theta=\Omega \in \mathbf{R}$. On the other hand, in the latter case, $\Omega \in \mathbf{R}^{2 \times 2}$ is given of the form $\Omega=\theta_{\mathrm{w}} I_{2}\left(\theta_{\mathrm{w}} \in \mathbf{R}\right)$ and two of the eigenvalues of $M_{0}+\theta M_{1}$ that form a complex conjugate pair go across $j \mathbf{R} \backslash\{0\}$ at $\theta=\theta_{\mathrm{w}}$.

2) Robust Stability Analysis: Let us consider the problem (12) for the following matrix pair discussed in [10]:

$$
M_{0}=\left[\begin{array}{rrr}
-4 & 2 & -2 \\
5 & -6 & 1 \\
-2 & 2 & -7
\end{array}\right], \quad M_{1}=\left[\begin{array}{rrr}
-5 & -3 & -13 \\
-5 & 0 & 0 \\
10 & 13 & 16
\end{array}\right]
$$

Here, the matrix $M_{0}$ is Hurwitz stable. To solve this problem, we first carried out a bisection search over $\delta$ in (11) with $N=1$, which terminated at $\delta_{1}=0.8026$. However, the resulting $\mathcal{H}$ does not satisfy (4) and thus we can conclude nothing. With this in mind, we next let $N=3$. Then, we confirmed that the bisection search terminates at $\delta=1.1059$ with $\operatorname{rank}(\overline{\mathcal{H}})=$ $\operatorname{rank}(\mathcal{H})=1$, which ensures the exactness of the computed stability margin. Exactly the same result was obtained even when we consider the dual problem corresponding to $N=2$.

Since $M_{0}$ is Hurwitz stable in this case, we can also apply the method in [2] to compute $\delta_{\max }$ exactly, which turns out to be 1.1059. In addition, it was shown rigorously in [10] that for the exact stability margin computation, the degree of the multiplier needs to be at least two. The present results are surely consistent with these preceding results.

3) Robust $H_{\infty}$ Performance Analysis: Let us consider the linear system described by

$$
G(s)=\frac{\omega_{1}^{2} \omega_{2}^{2}}{\left(s^{2}+2 \zeta_{1} \omega_{1} s+\omega_{1}^{2}\right)\left(s^{2}+2 \zeta_{2} \omega_{2} s+\omega_{2}^{2}\right)}
$$

where $\zeta_{1}=0.2, \omega_{1}=1.0, \zeta_{2}=1.0$ and $\omega_{2}=3.0$. The $H_{\infty}$ norm of $G(s)$ is $\|G\|_{\infty}=2.3167$. Here we consider the $H_{\infty}$ performance margin analysis problem with respect to a perturbation on $\zeta_{2}$. To this end, let us denote by $G_{\theta}(s)$ the system obtained by replacing $\zeta_{2}$ in $G(s)$ by $\zeta_{2}(1+\theta)$. Then, for given $\gamma(>2.3167)$, the problem here is to compute $\delta_{\max }:=\max \delta$ such that $\left\|G_{\theta}\right\|_{\infty}<\gamma$ holds for all $\theta \in(-\delta, \delta)$.

It should be noted that the controllability canonical realization of $G_{\theta}(s)$ can be written in the form of $G_{\theta}(s)=\left\{A_{0}+\right.$ $\left.\theta A_{1}, B, C, 0\right\}\left(A_{0}, A_{1} \in \mathbf{R}^{4 \times 4}\right)$. Thus, by using the idea of Hamiltonian eigenvalue tests [12], [35], the present robust $H_{\infty}$ 
TABLE I

ROBUST $H_{\infty}$ PERFORMANCE MARGIN ANALYSIS

\begin{tabular}{|c|c|c|c|c|}
\hline$\gamma$ & $\delta_{\max }$ & $\theta_{\mathrm{w}}$ & $\omega_{\mathrm{w}}$ & $\left\|G_{\theta_{\mathrm{w}}}\right\|_{\infty}$ \\
\hline 3.0 & 0.2104 & -0.2104 & 0.7506 & 3.0000 \\
4.0 & 0.3889 & -0.3889 & 0.5803 & 4.0000 \\
5.0 & 0.4960 & -0.4960 & 0.4781 & 5.0000 \\
6.0 & 0.5675 & -0.5675 & 0.4100 & 6.0000 \\
7.0 & 0.6185 & -0.6185 & 0.3614 & 6.9999 \\
\hline
\end{tabular}

performance analysis problem reduces to Problem 1 where

$$
M_{0}=\left[\begin{array}{cc}
A_{0} & \frac{B B^{T}}{\gamma^{2}} \\
-C^{T} C & -A_{0}^{T}
\end{array}\right], \quad M_{1}=\left[\begin{array}{cc}
A_{1} & 0 \\
0 & -A_{1}^{T}
\end{array}\right] .
$$

By means of this problem transformation, we solved (11) for $\gamma \in\{3.0,4.0,5.0,6.0,7.0\}$ by letting $N=3$. More precisely, we carried out a bisection search over $\delta$ in (11) to detect $\delta_{\max }$ for each $\gamma$. Then, for every tested $\gamma$, the condition $\operatorname{rank}\left(\mathcal{H}_{0}\right)=$ $\operatorname{rank}(\mathcal{H})=2$ is satisfied at the minimal value of $\delta$. The resulting $\Omega$ is given of the form $\Omega=\theta_{\mathrm{w}} I_{2}$. In Table I, we show the computed performance margin $\delta_{\max }$, the worst case perturbation $\theta_{\mathrm{w}}$ and $\left\|G_{\theta_{\mathrm{w}}}\right\|_{\infty}$. We also show the worst case angular frequency $\omega_{\mathrm{w}}$ satisfying $\left\|G_{\theta_{\mathrm{w}}}\left(j \omega_{\mathrm{w}}\right)\right\|=\left\|G_{\theta_{\mathrm{w}}}\right\|_{\infty}$, which can readily be obtained by constructing $\Gamma$ from (6) and computing its eigenvalues. In this way, we have achieved exact analysis in the case where $N=3$. We also confirmed that, even in the case where $N=1$, exactly the same results can be obtained except for $\gamma=3.0$.

\section{Connections to Polynomial Matrix Inequality Formulation and Known Exactness Verification Tests}

Since a matrix $A \in \mathbf{R}^{n \times n}$ satisfies $\{\lambda(A) \cap j \mathbf{R}\} \neq \phi$ if and only if there exists $X \in \mathbf{P}_{n} \backslash\{0\}$ such that $\operatorname{He}\{A X\}=0$, it is also possible to formulate Problem 1 as a polynomial matrix inequality (PMI) problem given in the following:

$$
\begin{aligned}
& \text { Find } \mathcal{H}_{0} \in \mathbf{P}_{n} \backslash\{0\} \text { and } \theta \in \Theta_{\delta} \text { such that } \\
& \operatorname{He}\left\{\left(M_{0}+\theta M_{1}\right) \mathcal{H}_{0}\right\}=0 .
\end{aligned}
$$

We note that general PMI problems are thoroughly investigated in [16], where a hierarchy of LMI relaxations with the theoretical guarantee of convergence is suggested. Since (15) involves $\mathcal{H}_{0}$ with $N_{s}:=n(n+1) / 2$ scalar variables, however, it is impracticable to apply the method in [16] directly to (15). To illustrate this point, let us consider again the 100 problem instances discussed in II-C1 where $n=5$. Following [16], we carried out the first LMI relaxation for (15) and solved an LMI with respect to the moment matrix variable $\mathcal{M}_{1}$, which is symmetric of the size $2+N_{s}=17$. Then, it turned out that $\operatorname{rank}\left(\mathcal{M}_{1}\right)=1$ in one case and $\operatorname{rank}\left(\mathcal{M}_{1}\right)=2$ in the rest 99 cases, which implies that we can detect the worst case perturbation only for one problem instance. The average CPU time was 0.42 [sec]. To obtain more satisfactory results, we are lead to apply the second LMI relaxation. However, its computational burden becomes prohibitive since we need to deal with the moment matrix $\mathcal{M}_{2}$ of the size $\left(N_{s}+3\right)\left(N_{s}+2\right) / 2=153$.

It is clear that (3) gives another LMI relaxation for the PMI (15). Similarly to [16], the computational burden associated with (3) of course grows up if we increase $N$ but its growth is rather moderate. In particular, in all tested numerical experiments, we can successfully detect the worst case perturbation under small $N$, say, $N=1,2$ or 3 .
Once we have revealed that (3) is an LMI relaxation for (15), it is obvious that another exactness verification test for (3), which goes beyond the rank-one exactness principle, can be given as follows: $\exists \theta \in \Theta_{\delta}$ such that $\mathcal{H}_{i}=\theta^{i} \mathcal{H}_{0}(i=1, \ldots, N+1)$. This can be restated equivalently as a linear problem of the form

$$
\exists \theta \in \Theta_{\delta} \text { such that }\left[I_{(N+1) n / 2} \quad \theta I_{(N+1) n / 2}\right] J_{n, N}^{T} \mathcal{H}=0 .
$$

In [30], [31], an exactness verification test for completely general robust LMI problems is suggested and it does apply to (3). Even though the discussion in [30], [31] does not clearly mention the structural property of dual solutions, we can confirm that the exactness verification test to (3), in the spirit of [30], [31], can be given as (16). Obviously, if (16) holds then (4) holds. It follows that we have given a more general exactness verification test dedicated to the robustness analysis of linear systems. This generalization is surely meaningful, and we have already observed the effectiveness of (4) over (16) in II-C1 (ex., for (10), the condition (16) fails whereas (4) does hold).

To summarize this section, we confirmed that the exactness verification test in Theorem 1 in conjunction with the trace-minimization heuristic and bisection search works effectively in practice. The discussion above also supports the validity of our approach in comparison with the existing studies. These observations strongly motivate us to further extend the results in Theorem 1 . The following question naturally arises: is it possible to obtain consistent results when $M(\theta)$ depends affinely or rationally on multiple uncertain parameter $\theta \in \Theta_{\delta}^{L}:=[-\delta, \delta]^{L}$ ?

It turns out from subsequent discussions that we can provide an affirmative answer to this question. More specifically, we reveal that the form of the rank-condition for the exactness verification varies according to the structure of $M(\theta)$ and the number of uncertain parameters. This unfortunately prevents us from constructing a unified treatment. Therefore we tackle each case separately in the sequel.

\section{RATIONAL PARAMETER-DEPENDENCE ON SINGLE UNCERTAIN PARAMETER}

Let us consider a rational function $M(\theta): \mathbf{R} \rightarrow \mathbf{R}^{n \times n}$ without pole at zero. This implies that $M(\theta)$ admits the linear fractional transformation (LFT) representation of the form

$$
M(\theta)=M_{11}+M_{12}\left(I_{l}-\theta M_{22}\right)^{-1} \theta M_{21} \text {. }
$$

The problem we posed here is to determine whether $I-\theta M_{22}$ is nonsingular (i.e., the LFT is well-posed) and $\{\lambda(M(\theta)) \cap$ $j \mathbf{R}\}=\phi$ holds for all $\theta \in \Theta_{\delta}$.

Since $\Theta_{\delta}$ is compact, we see from the idea of LMI dilation [9] that the above two requirements hold if and only if there exists $P(\theta): \Theta_{\delta} \rightarrow \mathbf{S}_{n}$, which can be taken as a polynomial [6], such that

$$
\begin{aligned}
& {\left[\begin{array}{cc}
\operatorname{He}\left\{P(\theta) M_{11}\right\} & -P(\theta) M_{12} \\
-M_{12}^{T} P(\theta) & 0
\end{array}\right]} \\
& \quad-\left[\begin{array}{c}
M_{21}^{T} \theta \\
I_{l}-M_{22}^{T} \theta
\end{array}\right]\left[\begin{array}{c}
M_{21}^{T} \theta \\
I_{l}-M_{22}^{T} \theta
\end{array}\right]^{T} \prec 0 \quad \forall \theta \in \Theta_{\delta} .
\end{aligned}
$$

This auxiliary step to derive (17) from the standard $\operatorname{He}\{P(\theta) M(\theta)\} \prec 0$ is crucial, which enables us to deal with the two independent requirements in a unified fashion 
(rather than assuming the well-posedness as usual). In addition, the above problem reformulation allows us to decide the degree of the left-hand side of (17) depending solely upon the degree of $P(\theta)$, irrespectively of the form of the rational function $M(\theta)$. Similar observation can be found in [30], where the full-block $\mathcal{S}$-procedure [18], [20], [28] plays a key role.

Once we have obtained (17), it is straightforward to derive numerically verifiable (but conservative in general) finite-dimensional LMIs by restricting $P(\theta)$ to be a polynomial of finite degree. In particular, by taking the dual of the LMI resulting from $(D, G)$ scaling, the next result follows. Here we only state the results corresponding to the multipliers of odd degree for the same reasons as before.

Theorem 3: For given positive odd number $N$, let us consider the following LMI-LME condition with respect to $\mathcal{H} \in$ $\mathbf{S}_{(N+3)(n+l) / 2}$ that has the block-Hankel matrix structure:

Find $\mathcal{H} \in \mathbf{S}_{(N+3)(n+l) / 2} \backslash\{0\}$ such that

$$
\begin{aligned}
& \mathcal{H}=\left[\begin{array}{cccc}
\mathcal{H}_{0} & \mathcal{H}_{1} & \ldots & \mathcal{H}_{(N+1) / 2} \\
\mathcal{H}_{1} & \therefore & \therefore & \vdots \\
\vdots & \therefore & \therefore & \mathcal{H}_{N} \\
\mathcal{H}_{(N+1) / 2} & \cdots & \dot{\mathcal{H}}_{N} & \mathcal{H}_{N+1}
\end{array}\right] \succeq 0 \\
& \delta^{2} \overline{\mathcal{H}}-\underline{\mathcal{H}} \succeq 0 \\
& \overline{\mathcal{H}}:=\langle\mathcal{H}\rangle^{(N+1)(n+l) / 2}, \quad \underline{\mathcal{H}}:=\langle\mathcal{H}\rangle_{(N+1)(n+l) / 2} \\
& \operatorname{He}\left\{\left[M_{11}-M_{12}\right] \mathcal{H}_{j}\left[I_{n} 0_{n, l}\right]^{T}\right\}=0(j=0, \ldots, N) \\
& \mathcal{W}\left[\begin{array}{ll}
\mathcal{H}_{0} & \mathcal{H}_{1} \\
\mathcal{H}_{1} & \mathcal{H}_{2}
\end{array}\right] \mathcal{W}^{T}=0 \\
& \mathcal{W}:=\left[\begin{array}{llll}
0_{l, n} & I_{l} & M_{21} & -M_{22}
\end{array}\right]
\end{aligned}
$$

i) Suppose (18) is infeasible. Then, $I-\theta M_{22}$ is nonsingular and $\{\lambda(M(\theta)) \cap j \mathbf{R}\}=\phi$ holds for all $\theta \in \Theta_{\delta}$.

ii) Suppose (18) is feasible and has a solution $\mathcal{H}$. Then, if

$$
\begin{aligned}
\operatorname{rank}\left(V_{N} \overline{\mathcal{H}} V_{N}^{T}\right) & =\operatorname{rank}(\mathcal{H}) \\
V_{N} & :=I_{(N+1) / 2} \otimes\left[\begin{array}{ll}
I_{n} & 0_{n, l}
\end{array}\right]
\end{aligned}
$$

there exists $\theta_{\mathrm{w}} \in \Theta_{\delta}$ such that $\operatorname{det}\left(I-\theta_{\mathrm{w}} M_{22}\right)=0$ or otherwise $\left\{\lambda\left(M\left(\theta_{\mathrm{w}}\right)\right) \cap j \mathbf{R}\right\} \neq \phi$. More precisely, if we denote the full-rank factorization of $\mathcal{H}$ by $\mathcal{H}=H H^{T}\left(H \in \mathbf{R}^{(N+3)(n+l) / 2 \times m}\right)$ and define $\bar{H}:=\lceil H\rfloor^{(N+1)(n+l) / 2}, \underline{H}:=\lceil H\rfloor_{(N+1)(n+l) / 2}$ and $\Omega:=\left(V_{N} \bar{H}\right)^{\dagger} V_{N} \underline{H}$, then $\Omega \in \mathbf{S}_{m}$ and $\lambda(\Omega) \subset \Theta_{\delta}$. In addition, we have $\operatorname{det}\left(I-\lambda_{k}(\Omega) M_{22}\right)=0$ or otherwise $\left\{\lambda\left(M\left(\lambda_{k}(\Omega)\right) \cap j \mathbf{R}\right\} \neq \phi\right.$ for all $k=1, \ldots, m$.

iii) If there exists $\theta_{\mathrm{w}} \in \Theta_{\delta}$, then there exists $\mathcal{H} \in$ $\mathbf{S}_{(N+3)(n+l) / 2} \backslash\{0\}$ such that (18) and (19) hold.

Proof: See Appendix section.

In addition to the assertions i), ii), and iii) in the above theorem, we can clarify mutual connections among the solutions of (18) for different degree $N$ as in the case of affine parameter dependence. It is also straightforward to verify that the rank condition (19) becomes more likely to be satisfied by increasing $N$. We omit the detailed discussions around these points, and move on to the comparison among Theorems 1 and 3 .

We note that the required rank condition (19) in Theorem 3 is slightly different from (4). Namely, the size of the matrix $V_{N} \overline{\mathcal{H}} V_{N}^{T}$ involved in the rank condition is $(N+1) n / 2$ and reduced from that of $\overline{\mathcal{H}}$ by $(N+1) l / 2$. This stems from the LMI dilation where we dilate the original robust LMI of the size $n$ to $n+l$ so that the difficulty arising from rational parameter dependence can be circumvented. It follows that we cannot conclude the exactness if we only have $\operatorname{rank}(\overline{\mathcal{H}})=\operatorname{rank}(\mathcal{H})$. However, we emphasize that this rank condition still works effectively to detect the worst case perturbations. A salient feature is that, if this rank condition holds, then the matrix $\Omega \in \mathbf{S}_{m}$ satisfying $\underline{H}=\bar{H} \Omega$ is uniquely determined by $\Omega=\bar{H}^{\dagger} \underline{H}$. This leads us to the next theorem that provides a more general exactness verification test than (19).

Theorem 4: Suppose (18) is feasible and has a solution $\mathcal{H}$ satisfying $\operatorname{rank}(\overline{\mathcal{H}})=\operatorname{rank}(\mathcal{H})=m$. Let us denote the fullrank factorization of $\mathcal{H}$ by $\mathcal{H}=H H^{T}$ and define $\bar{H}$ and $\underline{H}$ as in Theorem 3. Then, if there exists $\Gamma \in \mathbf{S k}_{m}$ such that

$$
\begin{aligned}
{\left[\begin{array}{ll}
M_{11} & M_{12}
\end{array}\right]\lceil H]^{n+l} } & =\lceil H\rfloor^{n} \Gamma \\
\Gamma \Omega & =\Omega \Gamma, \quad \Omega:=\bar{H}^{\dagger} \underline{H} \in \mathbf{S}_{m}
\end{aligned}
$$

this $\Omega$ satisfies exactly the same assertions in ii) of Theorem 3 .

Proof: See Appendix section.

It is obvious that if the rank condition (19) holds, then the condition $\operatorname{rank}(\overline{\mathcal{H}})=\operatorname{rank}(\mathcal{H})$ in Theorem 4 is automatically satisfied. Moreover, from the proof of Theorems 3 and 4, we see that (19) provides a sufficient condition to ensure the existence of $\Gamma \in \mathbf{S k}_{m}$ satisfying (20). It follows that the exactness verification test in Theorem 4 is surely more general than that of Theorem 3. We stress that the problem to find $\Gamma \in \mathbf{S k}_{m}$ satisfying (20) is a linear problem and thus can be solved efficiently.

By carrying out extensive numerical experiments, we can confirm that these two theorems work effectively in practice. Even for those problem instances where Theorem 3 fails, it is still possible to conclude the exactness and extract the worst case perturbations by means of Theorem 4 (see related illustrative examples in Section IV-B).

\section{Multiple Uncertain Parameter CASes}

Based on the preceding detailed analysis on the single uncertain parameter cases, we now move on to the analysis of multiple uncertain parameter cases. It turns out that the role of simultaneous diagonalizability property of commuting diagonalizable matrix family becomes more crucial when dealing with multiple uncertain parameters.

\section{A. Affine Parameter-Dependence}

For given $M_{i} \in \mathbf{R}^{n \times n}(i=0, \ldots, L)$, let us define $M_{\mathrm{a}}(\theta)$ : $\mathbf{R}^{L} \rightarrow \mathbf{R}^{n \times n}$ by $M_{\mathrm{a}}(\theta):=M_{0}+\sum_{i=1}^{L} \theta_{i} M_{i}$. This subsection evolves around the following problem:

Problem 2: For given $M_{i} \in \mathbf{R}^{n \times n}(i=0, \ldots, L)$ and $\delta>0$, determine whether $\left\{\lambda\left(M_{\mathrm{a}}(\theta)\right) \cap j \mathbf{R}\right\}=\phi$ holds for all $\theta \in$ $\Theta_{\delta}^{L}:=[-\delta, \delta]^{L}$.

As in the single-parameter cases, this problem can be assessed by seeking for a polynomial multiplier $P(\theta): \mathbf{R}^{L} \rightarrow \mathbf{S}_{n}$ that satisfies

$$
\operatorname{He}\left\{P(\theta) M_{\mathrm{a}}(\theta)\right\} \prec 0 \quad \forall \theta \in \Theta_{\delta}^{L} .
$$

Similarly, if we restrict our attention to the finite-degree polynomial approximation $P_{N}(\theta)$, the problem again amounts to finite-dimensional semi-infinite problem. In stark contrast with the single-parameter cases, however, it is hard to derive finite-dimensional LMIs to ensure the existence of the desired $P_{N}(\theta)$ exactly, even though effective asymptotically exact relaxations are suggested in [24], [30]-[32]. 
To cope with this difficult situation, we make another effort so that we can obtain exactness verification tests that are consistent with the single parameter cases. To this end, let us consider the existence condition of $P_{\mathrm{a}}(\theta)=P_{0}+\sum_{i=1}^{L} \theta_{i} P_{i}$ that satisfies

$$
\operatorname{He}\left\{P_{\mathrm{a}}(\theta) M_{\mathrm{a}}(\theta)\right\} \prec 0 \quad \forall \theta \in \Theta_{\delta}^{L} .
$$

Then, inspired by the $(D, G)$ scaling [23], we are lead to the following $\mathrm{LMI}$ relaxation:

Find $P_{i} \in \mathbf{S}_{n}(i=0, \ldots, L), D_{i} \in \mathbf{P}_{n}(i=1 \ldots, L)$, $G_{i j} \in \mathbf{S k}_{n}(i=0, \ldots, L-1, j=i+1, \ldots, L)$ such that

$$
\begin{aligned}
\text { He } & \left\{\left[\begin{array}{c}
P_{0} \\
P_{1} \\
P_{2} \\
\vdots \\
P_{L}
\end{array}\right]\left[\begin{array}{lllll}
M_{0} & M_{1} & M_{2} & \cdots & M_{L}
\end{array}\right]\right\} \\
& +\left[\begin{array}{ccccc}
\delta^{2} D & G_{01} & \cdots & \cdots & G_{0 L} \\
G_{01}^{T} & -D_{1} & G_{12} & \cdots & G_{1 L} \\
G_{02}^{T} & G_{12}^{T} & -D_{2} & \ddots & \vdots \\
\vdots & \vdots & \ddots & \ddots & G_{L-1, L} \\
G_{0 L}^{T} & G_{1 L}^{T} & \cdots & G_{L-1, L}^{T} & -D_{L}
\end{array}\right] \prec 0
\end{aligned}
$$

where $D=\sum_{i=1}^{L} D_{i}$. It can be easily verified that this LMI is surely a sufficient condition for the existence of the desired $P_{\mathrm{a}}(\theta)$. Indeed, if (23) holds, then multiplying $\left[\begin{array}{lllll}I & \theta_{1} I & \theta_{2} I & \ldots & \theta_{L} I\end{array}\right]$ from left and its transpose from right, we have $\operatorname{He}\left\{P_{\mathrm{a}}(\theta) M_{\mathrm{a}}(\theta)\right\}+\sum_{i=1}^{L} D_{i}\left(\delta^{2}-\theta_{i}^{2}\right) \prec 0$ and hence (22) surely holds.

It should be noted that the second term in (23) with $G_{i j}(i \geq$ 1) replaced by zeros appears in $(D, G)$ scaling for repeated real scalar block problems [23]. In this standard $(D, G)$ scaling, the correlation between the parameters $\theta_{i}$ and $\theta_{j}$ is not taken into account due to its inherent nature of the scalings. Once we have noticed that $\theta_{i} \theta_{j} I$ is also a real scalar block, it is quite natural to introduce a new variable $G_{i j}(i \geq 1)$ and derive (23). Note that this rationale to introduce $G_{i j}(i \geq 1)$ can also be explained along the lines of matrix SOS relaxations for robust LMI problems recently developed in [32]. Concrete discussions on the connections to [15], [22], [26] and [16], [30]-[32] are given after stating the main result of this subsection.

We emphasize that the introduction of $G_{i j} \in \mathbf{S k}_{n}(i \geq 1)$ does not allow us to remove the conservatism of the associated LMI relaxation completely. Nevertheless, this modification is significant in our dual LMI approach. This can be clearly seen from the dual LMI problem of (23) given as follows:

Find $\mathcal{H} \in \mathbf{S}_{(L+1) n} \backslash\{0\}$ such that

$$
\begin{aligned}
& \mathcal{H}=\left[\begin{array}{ccccc}
\mathcal{H}_{00} & \mathcal{H}_{01} & \mathcal{H}_{02} & \ldots & \mathcal{H}_{0 L} \\
\mathcal{H}_{01} & \mathcal{H}_{11} & & & \vdots \\
\mathcal{H}_{02} & & \ddots & & \mathcal{H}_{L-2, L} \\
\vdots & & & \mathcal{H}_{L-1, L-1} & \mathcal{H}_{L-1, L} \\
\mathcal{H}_{0 L} & \ldots & \mathcal{H}_{L-2, L} & \mathcal{H}_{L-1, L} & \mathcal{H}_{L L}
\end{array}\right] \succeq 0 \\
& \delta^{2} \mathcal{H}_{00}-\mathcal{H}_{i i} \succeq 0(i=1, \ldots, L) \\
& \operatorname{He}\left\{\left[M_{0} \ldots M_{L}\right] \mathcal{H} I_{(L+1) n, n}^{j}\right\}=0(j=1, \ldots, L+1) .
\end{aligned}
$$

Here, the matrix $I_{k n, n}^{j} \in \mathbf{R}^{k n \times n}$ is defined by $e_{j} \otimes I_{n}$ where $e_{j}$ is the $j$-th standard basis of the $k$-dimensional Euclidean space.
In the dual problem (24), we see that the dual variable $\mathcal{H}$ has the special block-matrix structure that is consistent with the moment matrix in [16], [22]. This is achieved by introducing $G_{i j} \in \mathbf{S k}_{n}(i \geq 1)$, which enforces symmetricity on $\mathcal{H}_{i j}(i=$ $1, \ldots, L, j=i+1, \ldots, L)$ so that the block-moment matrix structure of $\mathcal{H}$ has been completed. This fact, i.e., we can enforce the block-moment matrix structure on the dual variable $\mathcal{H}$, turns out to be the core to derive the desired rank condition for the exactness verification.

\section{Theorem 5:}

i) Suppose (24) is infeasible. Then, $\left\{\lambda\left(M_{\mathrm{a}}(\theta)\right) \cap j \mathbf{R}\right\}=\phi$ holds for all $\theta \in \Theta_{\delta}^{l}$.

ii) Suppose (24) is feasible and has a solution $\mathcal{H}$. Then, if

$$
\operatorname{rank}\left(\mathcal{H}_{00}\right)=\operatorname{rank}(\mathcal{H})
$$

there exists $\theta_{\mathrm{w}} \in \Theta_{\delta}^{L}$ such that $\left\{\lambda\left(M_{\mathrm{a}}\left(\theta_{\mathrm{w}}\right)\right) \cap j \mathbf{R}\right\} \neq \phi$. More precisely, if we denote the full-rank factorization of $\mathcal{H}$ by

$$
\mathcal{H}=\left[\begin{array}{c}
H_{0} \\
\vdots \\
H_{L}
\end{array}\right]\left[\begin{array}{c}
H_{0} \\
\vdots \\
H_{L}
\end{array}\right]^{T}, \quad H_{i} \in \mathbf{R}^{n \times m}(i=0, \ldots, L)
$$

and define $\Omega_{i}:=H_{0}^{\dagger} H_{i}(i=1, \ldots, L)$, then these matrices satisfy $\Omega_{i} \in \mathbf{S}_{m}, \lambda\left(\Omega_{i}\right) \subset \Theta_{\delta}(i=1, \ldots, L)$ and share all eigenvectors $u_{k} \in \mathbf{C}^{m}(k=1, \ldots, m)$ in common. In addition, if we denote by $\lambda_{k}\left(\Omega_{i}\right)$ the eigenvalue of $\Omega_{i}$ corresponding to the common eigenvector $u_{k}$, we have $\left\{\lambda\left(M_{0}+\sum_{i=1}^{L} \lambda_{k}\left(\Omega_{i}\right) M_{i}\right) \cap j \mathbf{R}\right\} \neq \phi$ for all $k=1, \ldots, m$.

iii) If there exists $\theta_{\mathrm{w}} \in \Theta_{\delta}^{L}$, then there exists $\mathcal{H} \in \mathbf{S}_{(L+1) n} \backslash$ $\{0\}$ such that (24) and (25) hold.

The result i) follows from strict alternative, while the result iii) can be proved via similar arguments to that of Theorem 1 . Thus, in the following, we will give the proof for ii), which states that if (25) is satisfied then we can easily extract the worst case perturbations by computing the eigenvalues of $\Omega_{i}(i=$ $1, \ldots, L)$ associated with the common eigenvectors. Since basic strategies for the proof are already established in the single parameter cases, the proof can be done in a streamlined fashion.

Proof: It is obvious from the block-moment matrix structure of $\mathcal{H}$ in (24) that $H_{i}(i=1, \ldots, L)$ in (26) satisfy $H_{i} H_{i}^{T} \preceq$ $\delta^{2} H_{0} H_{0}^{T}$ and $H_{0} H_{i}^{T}=H_{i} H_{0}^{T}$. Thus Lemma 2 verifies the existence of $\Omega_{i} \in \mathbf{S}_{m}$ with $\left\|\Omega_{i}\right\| \leq \delta$ satisfying $H_{i}=H_{0} \Omega_{i}(i=$ $1, \ldots, L)$. In particular, the block-moment matrix structure of $\mathcal{H}$ in (24) implies $H_{0} \Omega_{i} \Omega_{j} H_{0}^{T}=H_{0} \Omega_{j} \Omega_{i} H_{0}^{T}(i, j=1, \ldots, L)$. Since $H_{0}$ is of full-column rank from (25), we have $\Omega_{i} \Omega_{j}=$ $\Omega_{j} \Omega_{i}(i, j=1, \ldots, L)$. On the other hand, we see that the last $L+1$ equalities in (24) ensures the existence of $\Gamma, \Gamma_{i} \in$ $\mathbf{S k}_{m}(i=1, \ldots, L)$ such that

$$
\begin{aligned}
& {\left[\begin{array}{llll}
M_{0} & M_{1} & \ldots & M_{L}
\end{array}\right]\left[\begin{array}{c}
H_{0} \\
H_{0} \Omega_{1} \\
\vdots \\
H_{L} \Omega_{L}
\end{array}\right]=H_{0} \Gamma} \\
& {\left[\begin{array}{llll}
M_{0} & M_{1} & \ldots & M_{L}
\end{array}\right]\left[\begin{array}{c}
H_{0} \\
H_{0} \Omega_{1} \\
\vdots \\
H_{L} \Omega_{L}
\end{array}\right] \Omega_{i}=H_{0} \Gamma_{i}}
\end{aligned}
$$


where we applied Lemma 3 by noting again that $H_{0}$ is full-column rank. The above equalities clearly indicate $\Gamma \Omega_{i}=\Omega_{i} \Gamma(i=1, \ldots, L)$.

To summarize, we see that the family $\left\{\Gamma, \Omega_{1}, \ldots, \Omega_{L}\right\}$ is a commuting family satisfying (27). Thus, from Lemma 1 and proper modifications of the proof of Theorem 1, the results in ii) readily follow.

The results in Theorem 5 should be examined in comparison with the preceding results on polynomial optimization [15], [22], [26] and LMI relaxation for robust LMI problems [16], [29]-[32]. We summarize important observations in the following remark.

Remark 1:

1) As already noted, the derivation of (23) can also be explained along the line of the matrix SOS relaxation for robust LMI problems recently developed in [32]. More precisely, it was shown in [32] that (22) holds if and only if there exist $\varepsilon>0$ and SOS polynomial matrices $D_{i}(\theta)(i=$ $1, \ldots, L)$ such that

$$
-\operatorname{He}\left\{P_{\mathrm{a}}(\theta) M_{\mathrm{a}}(\theta)\right\}-\sum_{i=1}^{L} D_{i}(\theta)\left(\delta^{2}-\theta_{i}^{2}\right)-\varepsilon I_{n} \text { is SOS. }
$$

If we restrict the search of $D_{i}(\theta)(i=1, \ldots, L)$ to a finite-dimensional subspace, i.e., the space of SOS polynomial matrices with respect to a certain degree of monomial basis, we can reduce (28) into a finite-dimensional LMI problem [32]. The LMI (23) can be regarded as a particular case of this result where $D_{i}(\theta)=D_{i}(i=1, \ldots, L)$. Extension the results in theorem 5 to the cases where we employ higher-degree polynomial multipliers and SOS polynomial matrices $D_{i}(\theta)(i=1, \ldots, L)$ will be briefly discussed in Section IV-C.

2) The present approach is also closely related to [15], [22] and its matrix counterpart [16] that are known with the name of the theory of moments. Indeed, the structure of the dual variable $\mathcal{H}$ in (24) is surely consistent with the moment matrix described in [16], [22]. Contrary to the approach in [26], it has been shown in [15], [22] that the latter dual approach is effective for the exactness verification and optimal solution extraction in polynomial optimization. This is indeed the case when we deal with with PMIs [16]. In particular, it is reported in [15], [16], [22] that, in most problem instances, we can verify exactness via LMI relaxations of small degree. These facts strongly supports our present results.

3) The effectiveness of parameter-dependent multipliers to achieve less conservative results is well-recognized [5], [7]. The present dual LMI approach has clarified its significance more concretely. Namely, if we restrict $P(\theta)$ to be constant in (21), the corresponding dual LMI becomes (24) with the last $L+1$ equalities replaced by $\operatorname{He}\left\{\left[M_{0} \cdots M_{L}\right] \mathcal{H} I_{(L+1) n, n}^{1}\right\}=0$. This mere single equality does not allow us to derive (25), and the condition for the exactness verification degrades to

$$
\begin{aligned}
& \exists \theta \in \Theta_{\delta} \text { such that } \\
& \mathcal{H}_{i j}=\theta_{i} \theta_{j} \mathcal{H}_{00}(i=0, \ldots, L, j=i, \ldots, L) \\
& \text { where } \theta_{0}=1 .
\end{aligned}
$$

TABLE II

RESULTS FOR $n=5$

\begin{tabular}{|c|c|c|c|}
\hline$L$ & $N_{\text {feas }}$ & $N_{\text {rank }}$ & time [sec] \\
\hline 2 & 100 & 94 & 0.4480 \\
\hline 3 & 100 & 96 & 0.6430 \\
\hline 4 & 100 & 98 & 0.7910 \\
\hline 5 & 100 & 100 & 0.9596 \\
\hline 6 & 100 & 100 & 1.5854 \\
\hline 7 & 100 & 98 & 2.5476 \\
\hline
\end{tabular}

TABLE III

RESULTS FOR $L=2$

\begin{tabular}{|c|c|c|c|}
\hline$n$ & $N_{\text {feas }}$ & $N_{\text {rank }}$ & time [sec] \\
\hline 5 & 100 & 94 & 0.4480 \\
\hline 6 & 100 & 93 & 0.5060 \\
\hline 7 & 100 & 91 & 0.6456 \\
\hline 8 & 100 & 92 & 0.8524 \\
\hline 9 & 100 & 92 & 1.2741 \\
\hline 10 & 100 & 91 & 1.4647 \\
\hline
\end{tabular}

This condition is a simple generalization of (16) to the multiple parameter cases and thus closely related to the results in [30], [31]. Indeed, we can confirm that, by specializing the exactness verification test for general robust LMI problems shown in [30], [31] to (24), a linearized version of the condition (29) can be obtained. Obviously, if the condition (29) holds then (25) holds. It follows that, by means of the parameter-dependent multiplier $P_{\mathrm{a}}(\theta)$, we can obtain the exactness verification test (25) that is more general than (29). In relation to this observation, we note that employing parameter-dependent multiplier $P_{\mathrm{a}}(\theta)$ does not increase the decision variables in the dual LMI. It enforces more equality constraints on the dual variable $\mathcal{H}$ while loosens the rank condition for the exactness verification so that, if the dual solution exists, the worst case perturbation detection can be highly expected.

We now illustrate the effectiveness of Theorem 5 via numerical experiments.

1) Randomly Generated Matrices: For $M_{\mathrm{a}}(\theta)=M_{0}+$ $\sum_{i=1}^{L} \theta_{i} M_{i}$ with $M_{i}$ randomly generated in the same way as in Section II-C and $\delta=1$, we first examined how the suggested rank condition works effectively to detect the worst case perturbation. To obtain the results below, we solved the following problem expecting that the rank condition becomes more likely to be satisfied:

$$
\text { minimize } \operatorname{trace}(\mathcal{H}) \text { subject to }(24) \text { and } \operatorname{trace}\left(\mathcal{H}_{00}\right)=1 \text {. }
$$

Table II shows the results when we vary the number of the uncertain parameters $L$ for fixed matrix size $n=5$. On the other hand, Table III shows the results when we vary $n$ for fixed $L=2$. For each case, we generated 100 problem instances randomly, and in these tables $N_{\text {feas }}$ denotes the number that (30) is identified to be feasible, $N_{\text {rank }}$ the number that (25) is satisfied. We also show the average CPU time.

From these results, we could say that the suggested rank condition works effectively to ensure the exactness. On the other hand, it is hard to definitely conclude the computational complexity for solving (30) from these results. Nevertheless we could deduce that the computational complexity grows mildly with respect to both $L$ and $n$ (i.e., approximately linearly in $n$ ). 
For more concrete illustration, let us consider $M(\theta): \mathbf{R}^{4} \rightarrow$ $\mathbf{R}^{3 \times 3}$ described by

$$
M(\theta):=\left[\begin{array}{rrr}
-2.0+\theta_{1} & 0.4+\theta_{2} & -1.2+\theta_{1} \\
-1.2+\theta_{3} & -2.4+\theta_{4} & -0.4+\theta_{3} \\
-1.2+\theta_{1} & 0.4+\theta_{2} & -1.2+\theta_{1}
\end{array}\right] .
$$

The matrix $M(0)$ is Hurwitz stable. To assess the robust stability of this matrix, we first solve (30) with $\delta=1.0$. It turns out that (24) is identified to be feasible with CPU time 0.37 [sec], yielding $\operatorname{rank}\left(\mathcal{H}_{00}\right)=\operatorname{rank}(\mathcal{H})=2$ and the worst case perturbations

$$
\begin{aligned}
\theta_{\mathrm{w}, 1} & =\left[\begin{array}{llll}
1.0000 & 0.4928 & 0.8928 & 0.2000
\end{array}\right]^{T} \\
\theta_{\mathrm{w}, 2} & =\left[\begin{array}{llll}
1.0000 & -0.8928 & -0.4928 & 0.2000
\end{array}\right]^{T} .
\end{aligned}
$$

In this example, we can confirm that the resulting $\mathcal{H}$ does not satisfy (29). This fact clearly illustrates the effectiveness of the suggested exactness verification test (25) over (29).

Next, aiming at computing the exact stability margin $\delta_{\max }$ defined by

$$
\begin{aligned}
\delta_{\max } & :=\max \delta \quad \text { such that } \\
\{\lambda(M(\theta)) \cap j \mathbf{R}\} & =\phi \quad \forall \theta \in(-\delta, \delta)^{L}
\end{aligned}
$$

we carried out a bisection search over $\delta$ in (30). Then, the bisection search terminates at $\delta=0.8444$, yielding $\operatorname{rank}\left(\mathcal{H}_{00}\right)=$ $\operatorname{rank}(\mathcal{H})=2$ and the worst case perturbations

$$
\begin{aligned}
& \theta_{\mathrm{w}, 1}=\left[\begin{array}{llll}
0.8444 & 0.8444 & 0.8444 & 0.8444
\end{array}\right]^{T} \\
& \lambda\left(M\left(\theta_{\mathrm{w}, 1}\right)\right)=\{0.0000,-1.5333 \pm 0.4163 j\} \\
& \theta_{\mathrm{w}, 2}=\left[\begin{array}{llll}
0.8444 & -0.8444 & -0.8444 & 0.8444
\end{array}\right]^{T} \\
& \lambda\left(M\left(\theta_{\mathrm{w}, 2}\right)\right)=\{0.0000,-0.4481,-2.6186\} .
\end{aligned}
$$

Thus, we can conclude $\delta_{\max }=0.8444$ exactly. We note again the the condition (29) does not hold for the resulting $\mathcal{H}$, whereas (25) does hold.

2) Robust $H_{\infty}$ Performance Analysis: Let us consider the linear system described by $G_{\theta}(s)=\{A(\theta), B, C, 0\}$ where $B=C^{T}=\left[\begin{array}{lll}-0.3-0.6 & 0.4 & 0.3\end{array}\right]^{T}, A(\theta)=$

\begin{tabular}{|c|c|c|c|}
\hline$\gamma$ & $\delta_{\max }$ & $\theta_{\mathrm{w}}^{T}$ & $\left\|G_{\theta_{\mathrm{w}}}\right\|_{\infty}$ \\
\hline 1.0 & 0.0543 & {$\left[\begin{array}{lll}-0.0543 & 0.0543 & -0.0542\end{array}\right]$} & 1.0000 \\
\hline 2.0 & 0.1903 & {$\left[\begin{array}{lll}-0.1903 & -0.1903 & -0.1903]\end{array}\right.$} & 2.0000 \\
\hline 4.0 & 0.1995 & {$\left[\begin{array}{lll}-0.1839 & -0.1995 & -0.1995\end{array}\right]$} & 4.0003 \\
\hline 6.0 & 0.2012 & {$\left[\begin{array}{lll}-0.1831 & -0.2012 & -0.2012]\end{array}\right.$} & 6.0013 \\
\hline 8.0 & 0.2019 & 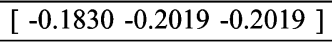 & 8.0027 \\
\hline 10.0 & 0.2023 & {$\left[\begin{array}{lll}-0.1830 & -0.2023 & -0.2023\end{array}\right]$} & 10.0045 \\
\hline 20.0 & 0.2030 & {$\left[\begin{array}{lll}-0.1831 & -0.2030 & -0.2030\end{array}\right]$} & 20.0328 \\
\hline$\infty$ & 0.2036 & {$\left[\begin{array}{lll}-0.1835 & -0.2036 & -0.2036\end{array}\right]$} & \\
\hline
\end{tabular}
$A_{0}+\sum_{i=1}^{3} \theta_{i} A_{i}$ and

$$
\begin{aligned}
A_{0} & =\left[\begin{array}{rrrr}
-0.8 & 0.5 & -0.6 & -0.3 \\
0.0 & -0.4 & 0.1 & -0.1 \\
0.1 & 0.1 & -0.5 & -0.2 \\
0.2 & 0.5 & -0.4 & -0.5
\end{array}\right] \\
A_{1} & =\left[\begin{array}{rrrr}
-0.9 & 0.5 & -0.5 & -0.3 \\
-0.2 & 0.1 & -0.1 & -0.6 \\
-0.4 & 0.3 & 0.5 & -0.1 \\
0.6 & 0.5 & -0.3 & 0.2
\end{array}\right] \\
A_{2} & =\left[\begin{array}{lrrr}
0.1 & -0.3 & -0.1 & 0.3 \\
0.9 & -0.6 & -0.7 & -0.8 \\
0.2 & -0.4 & -0.4 & -0.2 \\
0.1 & -0.3 & 0.0 & -0.3
\end{array}\right] \\
A_{3} & =\left[\begin{array}{rrrr}
-0.2 & 0.1 & 0.0 & 0.3 \\
0.3 & -0.2 & -0.6 & -0.5 \\
0.2 & -0.6 & 0.4 & 0.4 \\
0.8 & -0.1 & -0.3 & 0.2
\end{array}\right] .
\end{aligned}
$$

We can confirm that $A_{0}$ is Hurwitz stable and the $H_{\infty}$ norm of the nominal system (i.e., $G_{0}(s)$ ) is 0.9411 . Our interest here is to compute the robust $H_{\infty}$ performance margin $\delta_{\max }:=\max \delta$ such that $\left\|G_{\theta}\right\|_{\infty}<\gamma$ holds for all $\theta \in(-\delta, \delta)^{3}$.
TABLE IV

RoBust $H_{\infty}$ PeRformance ANAlysis

Solving this problem by constructing a Hamiltonian matrix and carrying out a bisection search over $\delta$ in (30), we obtained the results summarized in Table IV. The bisection search terminates, yielding $\operatorname{rank}\left(\mathcal{H}_{00}\right)=\operatorname{rank}(\mathcal{H})=2$ for $\gamma=1$ and $\operatorname{rank}\left(\mathcal{H}_{00}\right)=\operatorname{rank}(\mathcal{H})=1$ for the other cases. Thus the suggested rank condition works fine to detect the worst case perturbation and to confirm the exactness of the resulting performance margin. Note that in the table, the results in the row $\gamma=\infty$ are obtained when we simply carried out robust stability margin analysis. It can be seen that the worst case perturbation $\theta_{\mathrm{w}}$ converges to the destabilizing perturbation by increasing $\gamma$.

\section{B. Rational Parameter-Dependence}

We next extend the preceding results to the case of rational parameter-dependence. Let us consider the rational function $M_{\mathrm{r}}(\theta): \mathbf{R}^{L} \rightarrow \mathbf{R}^{n \times n}$ described by the LFT form $M_{\mathrm{r}}(\theta)=M_{11}+M_{12}\left(I_{l}-\Delta(\theta) M_{22}\right)^{-1} \Delta(\theta) M_{21}$. We assume that $\Delta(\theta): \mathbf{R}^{L} \rightarrow \mathbf{R}^{l \times l}(l \geq L)$ is of the form $\Delta(\theta)=\sum_{i=1}^{L} \theta_{i} E_{i}$, where $E_{i} \in \mathbf{R}^{l \times l} \backslash\{0\}(i=1, \ldots, L)$ are given diagonal matrices whose diagonal entries are zero or one and satisfy $\sum_{i=1}^{L} E_{i}=I_{l}$. This structure is commonly adopted in the community of control theory. As in Section III, the problem we posed here it to determine whether $I-\Delta(\theta) M_{22}$ is nonsingular and $\left\{\lambda\left(M_{\mathrm{r}}(\theta)\right) \cap j \mathbf{R}\right\}=\phi$ holds for all $\theta \in \Theta_{\delta}^{L}$.

Even though the description of $M_{\mathrm{r}}(\theta)$ becomes rather complicated, basic ideas of LMI dilation in Section III and (modified) $(D, G)$ scaling in Section IV-A can be applied to assess the existence of $P(\theta): \Theta_{\delta}^{L} \rightarrow \mathbf{S}_{n}$ such that $\operatorname{He}\left\{P(\theta) M_{\mathrm{r}}(\theta)\right\} \prec$ $0\left(\forall \theta \in \Theta_{\delta}^{L}\right)$. In particular, by employing $P_{\mathrm{a}}(\theta)=P_{0}+$ $\sum_{i=1}^{L} \theta_{i} P_{i}$, we can obtain the next two theorems.

Theorem 6: Let us consider the following LMI-LME with respect to $\mathcal{H} \in \mathbf{S}_{(L+1)(n+l)}$ :

Find $\mathcal{H} \in \mathbf{S}_{(L+1)(n+l)} \backslash\{0\}$ such that

$$
\begin{aligned}
& \mathcal{H}=\left[\begin{array}{ccccc}
\mathcal{H}_{00} & \mathcal{H}_{01} & \mathcal{H}_{02} & \ldots & \mathcal{H}_{0 L} \\
\mathcal{H}_{01} & \mathcal{H}_{11} & & & \vdots \\
\mathcal{H}_{02} & & \ddots & & \mathcal{H}_{L-2, L} \\
\vdots & & & \mathcal{H}_{L-1, L-1} & \mathcal{H}_{L-1, L} \\
\mathcal{H}_{0 L} & \ldots & \mathcal{H}_{L-2, L} & \mathcal{H}_{L-1, L} & \mathcal{H}_{L L}
\end{array}\right] \succeq 0 \\
& \delta^{2} \mathcal{H}_{00}-\mathcal{H}_{i i} \succeq 0(i=1, \ldots, L) \\
& \operatorname{He}\left\{\left[\begin{array}{ll}
M_{11}-M_{12}
\end{array}\right] \mathcal{H}_{0 j}\left[\begin{array}{ll}
I_{n} & 0_{n, l}
\end{array}\right]^{T}\right\}=0(j=0, \ldots, L) \\
& \mathcal{W H} \mathcal{W}^{T}=0 \\
& \mathcal{W}:=\left[0_{l, n} I_{l} E_{1} M_{21}-E_{1} M_{22}\right. \\
& \left.\ldots E_{L} M_{21}-E_{L} M_{22}\right] \text {. }
\end{aligned}
$$


i) Suppose (32) is infeasible. Then, $I-\Delta(\theta) M_{22}$ is nonsingular and $\left\{\lambda\left(M_{\mathrm{r}}(\theta)\right) \cap j \mathbf{R}\right\}=\phi$ holds for all $\theta \in \Theta_{\delta}^{L}$.

ii) Suppose (32) is feasible and has a solution $\mathcal{H}$. Then, if

$$
\operatorname{rank}\left(\left\langle\mathcal{H}_{00}\right\rangle^{n}\right)=\operatorname{rank}(\mathcal{H})
$$

there exists $\theta_{\mathrm{w}} \in \Theta_{\delta}^{L}$ such that $\operatorname{det}\left(I-\Delta\left(\theta_{\mathrm{w}}\right) M_{22}\right)=0$ or otherwise $\left\{\lambda\left(M_{\mathrm{r}}\left(\theta_{\mathrm{w}}\right)\right) \cap j \mathbf{R}\right\} \neq \phi$. More precisely, if we denote the full-rank factorization of $\mathcal{H}$ by

$$
\mathcal{H}=\left[\begin{array}{c}
H_{0} \\
\vdots \\
H_{L}
\end{array}\right]\left[\begin{array}{c}
H_{0} \\
\vdots \\
H_{L}
\end{array}\right]^{T}, \quad H_{i} \in \mathbf{R}^{(n+l) \times m}
$$

and define $\Omega_{i}:=\left(\left\lceil H_{0}\right\rfloor^{n}\right)^{\dagger}\left\lceil H_{i}\right\rfloor^{n}(i=1, \ldots, L)$, then these matrices satisfy $\Omega_{i} \in \mathbf{S}_{m}, \lambda\left(\Omega_{i}\right) \subset$ $\Theta_{\delta}(i=1, \ldots, L)$ and share all eigenvectors $u_{k} \in \mathbf{C}^{m}(k=1, \ldots, m)$ in common. In addition, if we denote by $\lambda_{k}\left(\Omega_{i}\right)$ the eigenvalues of $\Omega_{i}$ corresponding to the common eigenvector $u_{k}$, we have

$$
\operatorname{det}\left(I-\left(\sum_{i=1}^{L} \lambda_{k}\left(\Omega_{i}\right) E_{i}\right) M_{22}\right)=0
$$

or otherwise

$$
\begin{aligned}
\left\{\lambda \left(M_{11}+M_{12}(I\right.\right. & \left.-\left(\sum_{i=1}^{L} \lambda_{k}\left(\Omega_{i}\right) E_{i}\right) M_{22}\right)^{-1} \\
& \left.\left.\times\left(\sum_{i=1}^{L} \lambda_{k}\left(\Omega_{i}\right) E_{i}\right) M_{21}\right) \cap j \mathbf{R}\right\} \neq \phi
\end{aligned}
$$

for all $k=1, \ldots, m$.

iii) If there exists $\theta_{\mathrm{w}} \in \Theta_{\delta}$, then there exists $\mathcal{H} \in$ $\mathbf{S}_{(L+1)(n+l)} \backslash\{0\}$ such that (32) and (33) hold.

Theorem 7: Suppose (32) is feasible and has a solution $\mathcal{H}$ satisfying $\operatorname{rank}\left(\mathcal{H}_{00}\right)=\operatorname{rank}(\mathcal{H})=m$. Let us denote the fullrank factorization of $\mathcal{H}$ by (34). Then, if there exists $\Gamma \in \mathbf{S k}_{m}$ such that

$$
\begin{aligned}
& {\left[\begin{array}{ll}
M_{11} & M_{12}
\end{array}\right] H_{0}=\left\lceil H_{0}\right]^{n} \Gamma} \\
& \Gamma \Omega_{i}=\Omega_{i} \Gamma, \quad \Omega_{i}:=H_{0}^{\dagger} H_{i} \in \mathbf{S}_{m},(i=1, \ldots, L)
\end{aligned}
$$

these $\Omega_{i}(i=1, \ldots, L)$ satisfy exactly the same assertions in ii) of Theorem 6.

To avoid duplicated descriptions, we omit the formal proofs for these two theorems. Note that the proof for Theorem 6 can be established by applying those key ideas as LMI dilation, (modified) $(D, G)$ scaling and convex duality theory to derive the dual LMI (32). Then, by making good use of the simultaneous diagonalizability property of commuting diagonalizable matrices and following close arguments to the proof of Theorem 3 , we arrive at (33). It is also true that (33) provides a sufficient condition for the existence of $\Gamma \in \mathbf{S k}_{m}$ satisfying (35). Thus, the exactness verification test in Theorem 7 is more general than Theorem 6, and this generalization is surely meaningful as illustrated in the following numerical examples:

1) Robust Stability Analysis: Let us consider the linear system described by

$$
\dot{x}=M_{11} x+M_{12} w, \quad z=M_{21} x+M_{22} w, \quad w=\Delta(\theta) z
$$

$$
\begin{aligned}
& \text { where } \\
& G(s)=\left[\begin{array}{l|l}
M_{11} & M_{12} \\
\hline M_{21} & M_{22}
\end{array}\right]= \\
& {\left[\begin{array}{rrrrr|rrrr}
-0.5 & -0.5 & 0.0 & -0.3 & -0.3 & 0.5 & 0.0 & -0.3 & 0.0 \\
-0.6 & -1.0 & -0.6 & 0.2 & 0.8 & 0.0 & -0.3 & 0.8 & 0.0 \\
-0.3 & 0.3 & -0.7 & 0.6 & -0.6 & 0.9 & 0.1 & -0.1 & 0.0 \\
-0.6 & -0.4 & -0.6 & -0.6 & -0.1 & 0.0 & -0.4 & 0.2 & 0.0 \\
-0.4 & -0.4 & 0.2 & 0.3 & -1.1 & -0.1 & 0.4 & 0.3 & 0.0 \\
\hline-0.2 & -0.1 & 0.0 & -0.3 & -0.2 & 0.1 & 1.0 & 0.0 & 0.0 \\
-0.3 & -0.3 & 0.0 & 0.3 & -0.1 & -0.2 & 0.3 & 0.0 & 0.0 \\
0.0 & 0.0 & 0.0 & 0.0 & 0.0 & 0.0 & 0.0 & 0.0 & 1.0 \\
0.4 & 0.8 & -0.2 & -0.1 & 0.6 & 0.0 & 0.0 & 0.0 & 0.0
\end{array}\right]} \\
& \Delta(\theta)=\sum_{i=1}^{2} \theta_{i} E_{i}, \quad E_{1}=\operatorname{diag}\left(I_{2}, 0_{2}\right) \quad E_{2}=\operatorname{diag}\left(0_{2}, I_{2}\right) .
\end{aligned}
$$

By noting that $M_{11}$ is Hurwitz stable, our interest here is to compute the maximal value of $\delta$, denoted by $\delta_{\max }$, such that the feedback system (36) is well-posed and stable for all $\theta \in(-\delta, \delta)^{2}$. To this end, we carried out a bisection search over $\delta$ in the problem:

$$
\operatorname{minimize} \operatorname{trace}(\mathcal{H}) \text { subject to }(32) \text { and } \operatorname{trace}\left(\mathcal{H}_{00}\right)=1 \text {. }
$$

Then, the bisection search terminates at $\delta=0.9603$, yielding $\operatorname{rank}\left(\mathcal{H}_{00}\right)=\operatorname{rank}(\mathcal{H})=2$ whereas $\operatorname{rank}\left(\left\langle\mathcal{H}_{00}\right\rangle^{5}\right)=1$. Namely, the rank condition (33) in Theorem 6 does not hold. However, we can easily verify that the condition (35) in Theorem 7 is satisfied for $\Gamma=0$. Therefore, the computed stability margin is surely exact, and we can detect the worst case perturbations as $\left(\lambda_{1}\left(\Omega_{1}\right), \lambda_{1}\left(\Omega_{2}\right)\right)=(-0.2330,0.9603)$ and $\left(\lambda_{2}\left(\Omega_{1}\right), \lambda_{2}\left(\Omega_{2}\right)\right)=(-0.2330,-0.9603)$. We note that, in this example, the known condition (29) for the exactness verification also fails. As we have also observed in Sections II-C and IV-A, this typically occurs when there exist multiple worst case perturbations.

To examine the obtained result more carefully, we next computed the scaled $H_{\infty}$ norm of the system $G(s)$ with the scaling $D=\operatorname{diag}\left(D_{1}, D_{2}\right)\left(D_{1}, D_{2} \in \mathbf{P}_{2}\right)$, which turned out to be 1.4078. Namely, the lower bound of $\delta_{\max }$ computed from the standard scaled $H_{\infty}$ norm analysis is $1 / 1.4078=0.7103$. We see that this is far from the exact stability margin.

2) Robust $H_{\infty}$ Performance Analysis: Let us again consider the linear system (14). Here we consider the $H_{\infty}$ performance margin analysis problem with respect to the independent perturbations on $\zeta_{2}$ and $\omega_{2}$, which are denoted by $\zeta_{2}\left(1+\theta_{1}\right)$ and $\omega_{2}\left(1+\theta_{2}\right)$. To solve this problem, we construct a Hamiltonian matrix and converted it into the LFT form. We skip the technical details of this transformation; the size of the resulting matrices are $M_{11} \in \mathbf{R}^{8 \times 8}$ and $M_{22} \in \mathbf{R}^{12 \times 12}$.

By carrying out a bisection search over $\delta$ in (37), we obtained the results summarized in Table V. For every tested $\gamma$, the bisection search terminates with $\mathcal{H}$ satisfying $\operatorname{rank}\left(\left\langle\mathcal{H}_{00}\right\rangle^{8}\right)=$ $\operatorname{rank}(\mathcal{H})=2$ and $\Omega_{i}$ are given of the form $\Omega_{i}=\theta_{\mathrm{w} i} I_{2}(i=1$, 2). Thus, we can conclude that the computed performance margins are surely exact.

\section{Relaxation With Higher Degree Polynomial Multipliers}

Before closing this section, we briefly discuss the extension of the results in Theorems 5 and 6 along the line of the matrix SOS relaxations for robust LMI problems [32]. 
TABLE V

Robust $H_{\infty}$ Performance Margin ANalysis

\begin{tabular}{|c|c|c|c|}
\hline$\gamma$ & $\delta_{\max }$ & $\theta_{\mathrm{w}}^{T}$ & $\left\|G_{\theta_{\mathrm{w}}}\right\|_{\infty}$ \\
\hline 3.0 & 0.5174 & {$[-0.5174-0.5174]$} & 3.0000 \\
\hline 4.0 & 0.6610 & {$[-0.6610-0.6357]$} & 4.0000 \\
\hline 5.0 & 0.7354 & {$[-0.7354-0.6552]$} & 5.0000 \\
\hline 6.0 & 0.7821 & {$[-0.7821-0.6639]$} & 6.0000 \\
\hline 7.0 & 0.8145 & {$[-0.8145-0.6687]$} & 7.0000 \\
\hline
\end{tabular}

For concrete illustration, let us revisit the infinite-dimensional robust LMI (21). Its finite-dimensional counterpart, in the spirit of [32], is given as follows:

Find $P_{N_{1}}(\theta): \mathbf{R}^{L} \rightarrow \mathbf{S}_{n}$ and $D_{i}(\theta) \in \mathcal{D}_{N_{2}}(i=1, \ldots, L)$ such that

$$
-\operatorname{He}\left\{P_{N_{1}}(\theta) M_{\mathrm{a}}(\theta)\right\}-\sum_{i=1}^{L} D_{i}(\theta)\left(\delta^{2}-\theta_{i}^{2}\right)-\varepsilon I_{n} \text { is SOS. }
$$

Here, $P_{N_{1}}(\theta)$ is a polynomial matrix with monomials of total degree up to $N_{1}$. On the other hand, $\mathcal{D}_{N_{2}}$ is the space of SOS polynomial matrices with respect to the monomial basis constructed from all monomials of total degree up to $N_{2}$. For example, in the case where $L=2, N_{1}=2$ and $N_{2}=1$, those matrices $P_{2}(\theta)$ and $D_{i}(\theta)(i=1,2)$ are given in the following form:

$$
\begin{aligned}
& P_{2}(\theta)=\left[\begin{array}{c}
I_{n} \\
\theta_{1} I_{n} \\
\theta_{2} I_{n}
\end{array}\right]^{T} \widehat{P}_{2}\left[\begin{array}{c}
I_{n} \\
\theta_{1} I_{n} \\
\theta_{2} I_{n}
\end{array}\right], \quad \widehat{P}_{2} \in \mathbf{S}_{3 n} \\
& D_{i}(\theta)=\left[\begin{array}{c}
I_{n} \\
\theta_{1} I_{n} \\
\theta_{2} I_{n}
\end{array}\right]^{T} \widehat{D}_{i}\left[\begin{array}{c}
I_{n} \\
\theta_{1} I_{n} \\
\theta_{2} I_{n}
\end{array}\right], \quad \widehat{D}_{i} \in \mathbf{P}_{3 n} .
\end{aligned}
$$

The problem (38) can be reduced to a finite-dimensional LMI as we have done for $N_{1}=1$ and $N_{2}=0$ around (23) and (28). In addition, its dual LMI readily follows as in (24).

Our goal here is to derive an exactness verification test that is uniformly applicable to the general cases $N_{1} \geq 1$ and $N_{2} \geq 0$. To this end, we first clarify a common property of dual LMIs obtained in these cases. To see this, let us consider the case where $L=2, N_{1}=2$ and $N_{2}=1$ in (38). Then, the corresponding dual LMI is given as follows:

Find $\mathcal{Y} \in \mathbf{S}_{6 n} \backslash\{0\}$ such that

$$
\begin{gathered}
\mathcal{Y}=\left[\begin{array}{llllll}
\mathcal{Y}_{00} & \mathcal{Y}_{10} & \mathcal{Y}_{01} & \mathcal{Y}_{20} & \mathcal{Y}_{11} & \mathcal{Y}_{02} \\
\mathcal{Y}_{10} & \mathcal{Y}_{20} & \mathcal{Y}_{11} & \mathcal{Y}_{30} & \mathcal{Y}_{21} & \mathcal{Y}_{12} \\
\mathcal{Y}_{01} & \mathcal{Y}_{11} & \mathcal{Y}_{02} & \mathcal{Y}_{21} & \mathcal{Y}_{12} & \mathcal{Y}_{03} \\
\mathcal{Y}_{20} & \mathcal{Y}_{30} & \mathcal{Y}_{21} & \mathcal{Y}_{40} & \mathcal{Y}_{31} & \mathcal{Y}_{22} \\
\mathcal{Y}_{11} & \mathcal{Y}_{21} & \mathcal{Y}_{12} & \mathcal{Y}_{31} & \mathcal{Y}_{22} & \mathcal{Y}_{13} \\
\mathcal{Y}_{02} & \mathcal{Y}_{12} & \mathcal{Y}_{03} & \mathcal{Y}_{22} & \mathcal{Y}_{13} & \mathcal{Y}_{04}
\end{array}\right] \succeq 0 \\
\delta^{2}\left[\begin{array}{llll}
\mathcal{Y}_{00} & \mathcal{Y}_{10} & \mathcal{Y}_{01} \\
\mathcal{Y}_{10} & \mathcal{Y}_{20} & \mathcal{Y}_{11} \\
\mathcal{Y}_{01} & \mathcal{Y}_{11} & \mathcal{Y}_{02}
\end{array}\right]-\left[\begin{array}{lll}
\mathcal{Y}_{20} & \mathcal{Y}_{30} & \mathcal{Y}_{21} \\
\mathcal{Y}_{30} & \mathcal{Y}_{40} & \mathcal{Y}_{31} \\
\mathcal{Y}_{21} & \mathcal{Y}_{31} & \mathcal{Y}_{22}
\end{array}\right] \succeq 0, \\
\delta^{2}\left[\begin{array}{llll}
\mathcal{Y}_{00} & \mathcal{Y}_{10} & \mathcal{Y}_{01} \\
\mathcal{Y}_{10} & \mathcal{Y}_{20} & \mathcal{Y}_{11} \\
\mathcal{Y}_{01} & \mathcal{Y}_{11} & \mathcal{Y}_{02}
\end{array}\right]-\left[\begin{array}{llll}
\mathcal{Y}_{02} & \mathcal{Y}_{12} & \mathcal{Y}_{03} \\
\mathcal{Y}_{12} & \mathcal{Y}_{22} & \mathcal{Y}_{13} \\
\mathcal{Y}_{03} & \mathcal{Y}_{13} & \mathcal{Y}_{04}
\end{array}\right] \succeq 0 \\
\operatorname{He}\left\{\left[\begin{array}{llll}
M_{0} & M_{1} & M_{2} & 0_{n, 3 n}
\end{array}\right] \mathcal{Y}_{6 n, n}^{j}\right\}=0(j=1, \ldots, 6) .
\end{gathered}
$$

We omit the technical details for the derivation of this dual LMI. Note here that we have changed the way to put subscripts for each variables by following the description of the moment matrix in [16], [22].

From the structure of (39) and (24), it can be readily seen that if $\mathcal{Y}$ satisfies (39), then (24) is satisfied by $\mathcal{H}=\langle\mathcal{Y}\rangle^{3 n}$. More importantly, we can confirm that this property holds irrespective of $L, N_{1}$ and $N_{2}$, i.e., if we denote by $\mathcal{Y}$ the solution of the dual LMI corresponding to (38) with $N_{1} \geq 1$ and $N_{2} \geq 0$, then (24) is satisfied by

$$
\mathcal{H}=\langle\mathcal{Y}\rangle^{(L+1) n} .
$$

Thus, from (40) and Theorem 5, it is obvious that a sufficient rank condition that is uniformly applicable for the exactness verification is given by $\operatorname{rank}\left(\langle\mathcal{Y}\rangle^{n}\right)=\operatorname{rank}\left(\langle\mathcal{Y}\rangle^{(L+1) n}\right)$. In view of the fact that we can construct a hierarchy of LMI relaxations by increasing both $N_{1}$ and $N_{2}$ and the associated conservatism vanishes eventually [32], the result (40) should be of great importance.

Note however that the dual LMI corresponding to the higherdegree polynomial multipliers as in (39) is computationally demanding and its computational burden may become prohibitive even when we deal with rather small number of uncertain parameters. Since extensive numerical experiments in this paper illustrate that those (relatively concise) dual LMIs in Theorems 1 and 3 with $N=1$ and Theorems 5 and 6 corresponding to the first degree multiplier work effectively in most problem instances, we should first try these dual LMIs to extract exactness. From practical point of view, what is needed is to ensure the exactness of the analysis results at hand and in this respect, we believe that the results in this paper are useful certainly in a practical sense.

\section{CONCLUSION}

In this paper, we explored a dual LMI approach for robust performance analysis of linear systems affected by real parametric uncertainties. Particular emphasis has been laid upon the exactness verification and the worst case perturbation extraction. Observing analogies with the recent results on the polynomial optimization by means of the sum-of-squares relaxations, we have derived sound rank conditions for the exactness verification. Practical usefulness of the suggested rank condition has been fully illustrated through extensive numerical experiments.

\section{APPENDIX}

PROOF OF LEMMA 2: The assertion readily follows from Lemma 5 of [19]. To see this, let us note that the condition i) can be rewritten, equivalently as follows:

$$
G G^{T} \preceq(j F)(j F)^{*} \text { and }(j F) G^{T}+G(j F)^{*}=0 .
$$

It follows from Lemma 5 of [19] that the above conditions hold if and only if there exists a skew-symmetric Hermitian $\Upsilon$ with $\|\Upsilon\| \leq 1$ such that $G=j F \Upsilon$. By letting $\Omega=\operatorname{Re}(j \Upsilon)$, the conditions in ii) readily hold.

PROOF OF LEMMA 3: It is apparent that ii) implies i). To prove that i) implies ii), let us define the nonsingular matrix $T:=\left[F^{\dagger T} F^{\perp T}\right]^{T}$. Then, we have

$$
T\left(F G^{T}+G F^{T}\right) T^{T}=\left[\begin{array}{cc}
\operatorname{He}\left\{G^{T} F^{\dagger T}\right\} & G^{T} F^{\perp T} \\
F^{\perp} G & 0
\end{array}\right] .
$$


It follows from i) that $F^{\perp} G=0$ and thus we have $G=F \Gamma$ for some $\Gamma \in \mathbf{R}^{m \times m}$. Since i) holds and since $F$ is full-column rank, this $\Gamma$ obviously satisfy $\Gamma \in \mathbf{S k}_{m}$.

PROOF OF THEOREM 2: The first part is obvious from the block-Hankel matrix structure of the dual variable and hence we will prove the second part only. Obviously it suffices to show that the assertion holds for $N_{2}=N_{1}+2$.

From the underlying assumption and the proof of Theorem 1, there exist $\Gamma, \Gamma_{1} \in \mathbf{S k}_{m}$ that satisfy (6) and (7) for given $H_{0}$ and $\Omega$ for $N=N_{1}$. With this in mind, we show that $\mathcal{H}=$ $\mathcal{H}_{\mathrm{H}}^{N_{1}+2}\left(H_{0}, \Omega\right)$ surely satisfies (3) and (4) for $N=N_{1}+2$. Note that, since this matrix $\mathcal{H}$ has a conformable block-Hankel matrix structure and since (6) and (7) are satisfied, all we have to prove is that the next two equalities are satisfied:

$$
\begin{aligned}
& \operatorname{He}\left\{\left(M_{0} H_{0}+M_{1} H_{0} \Omega\right) \Omega^{N_{1}+1} H_{0}^{T}\right\}=0 \\
& \operatorname{He}\left\{\left(M_{0} H_{0}+M_{1} H_{0} \Omega\right) \Omega^{N_{1}+2} H_{0}^{T}\right\}=0 .
\end{aligned}
$$

To prove (41), recall that $\{\Omega, \Gamma\}$ is a commuting family. Hence, we have from (6) that

$$
\begin{aligned}
\left(M_{0} H_{0}+M_{1} H_{0} \Omega\right) \Omega^{N_{1}+1} & =H_{0} \Omega^{\left(N_{1}-1\right) / 2} \Gamma \Omega^{\left(N_{1}+3\right) / 2} \\
& =H_{0} \Omega^{\left(N_{1}+1\right) / 2} \Gamma \Omega^{\left(N_{1}+1\right) / 2}
\end{aligned}
$$

which clearly shows that (41) holds. On the other hand, to prove (42), it is important to note that (6) and (7) imply $\Gamma_{1} \Omega=\Gamma \Omega^{2}=$ $\Omega \Gamma \Omega \in \mathbf{S k}_{m}$. Thus, $\Gamma_{1} \Omega=\Omega \Gamma_{1}$ holds. It follows from (7) that

$$
\begin{aligned}
\left(M_{0} H_{0}+M_{1} H_{0} \Omega\right) \Omega^{N_{1}+2} & =H_{0} \Omega^{\left(N_{1}-1\right) / 2} \Gamma_{1} \Omega^{\left(N_{1}+3\right) / 2} \\
& =H_{0} \Omega^{\left(N_{1}+1\right) / 2} \Gamma_{1} \Omega^{\left(N_{1}+1\right) / 2}
\end{aligned}
$$

which implies (42). This completes the proof.

PROOF OF THEOREM 3: We first note that (18) corresponds to the dual of the LMI for the existence of $P_{N}(\theta)$ satisfying (17). Thus, the strict alternative ensures i). It should be elementary to confirm iii). Therefore only the proof for ii) is given in following.

As in the proof of Theorem 1, the block-Hankel matrix structure of $\mathcal{H}$ in (18) ensures the existence of $H_{0} \in \mathbf{R}^{(n+l) \times m}$ and $\Omega \in \mathbf{S}_{m}$ with $\|\Omega\| \leq \delta$ such that $H=\left[\begin{array}{llll}H_{0}^{T} & \Omega H_{0}^{T} & \cdots & \Omega^{(N+1) / 2} H_{0}^{T}\end{array}\right]^{T}$. This, as well as the last equality in (18) implies

$$
\left\lceil H_{0}\right\rfloor_{l}+M_{21}\left\lceil H_{0}\right\rfloor^{n} \Omega-M_{22}\left\lceil H_{0}\right\rfloor_{l} \Omega=0 .
$$

The $N+1$ equalities with respect to $\mathcal{H}_{j}(j=0, \ldots, N)$ in (18) can also be rewritten as

$$
\begin{gathered}
\operatorname{He}\left\{\left[\begin{array}{c}
{\left[M_{11}-M_{12}\right] H_{0}} \\
\vdots \\
{\left[M_{11}-M_{12}\right] H_{0} \Omega^{(N-1) / 2}}
\end{array}\right] \Omega^{k}\right. \\
\left.\times\left[\begin{array}{c}
\left\lceil H_{0}\right\rfloor^{n} \\
\vdots \\
\left\lceil H_{0}\right\rfloor^{n} \Omega^{(N-1) / 2}
\end{array}\right]^{T}\right\}=0 \quad(k=0,1) .
\end{gathered}
$$

Since $\left[\left(\left[H_{0}\right\rfloor^{n}\right)^{T} \ldots \Omega^{(N-1) / 2}\left(\left\lceil H_{0}\right\rfloor^{n}\right)^{T}\right]^{T}=V_{N} \bar{H}$ is of full-column rank from (19), it follows that there exists $\Gamma \in \mathbf{S k}_{m}$ such that

$$
\left[\begin{array}{ll}
M_{11} & -M_{12}
\end{array}\right] H_{0}=\left\lceil H_{0}\right\rfloor^{n} \Gamma, \quad \Gamma \Omega=\Omega \Gamma .
$$

To complete the proof, let us denote by $u_{k} \in \mathrm{C}^{m}(k=$ $1, \ldots, m)$ the common eigenvectors of $\Omega$ and $\Gamma$. Then, for each $u_{k}$, we see from (43) that

$$
\begin{aligned}
& \left(I-\lambda_{k}(\Omega) M_{22}\right)\left\lceil H_{0}\right\rfloor_{l} u_{k}+\lambda_{k}(\Omega) M_{21}\left\lceil H_{0}\right\rfloor^{n} u_{k}=0 \\
& \lambda_{k}(\Omega) \in \Theta_{\delta} .
\end{aligned}
$$

If $\left(I-\lambda_{k}(\Omega) M_{22}\right)$ is singular, we arrive at the desired conclusion. On the other hand, if $\left(I-\lambda_{k}(\Omega) M_{22}\right)$ is nonsingular, we have

$$
\left\lceil H_{0}\right\rfloor_{l} u_{k}=-\left(I-\lambda_{k}(\Omega) M_{22}\right)^{-1} \lambda_{k}(\Omega) M_{21}\left\lceil H_{0}\right\rfloor^{n} u_{k} .
$$

In addition, we see from the first equality in (44) that the following condition holds:

$$
\left[\begin{array}{ll}
M_{11} & -M_{12}
\end{array}\right] H_{0} u_{k}=\lambda_{\Gamma, k}\left\lceil H_{0}\right\rfloor^{n} u_{k}, \quad \lambda_{\Gamma, k} \in j \mathbf{R} .
$$

It follows from (45) and (46) that

$$
\begin{aligned}
\left(M_{11}+M_{12}\left(I-\lambda_{k}(\Omega) M_{22}\right)^{-1} \lambda_{k}(\Omega) M_{21}\right) & \left\lceil H_{0}\right\rfloor^{n} u_{k} \\
& =\lambda_{\Gamma, k}\left\lceil H_{0}\right\rfloor^{n} u_{k} .
\end{aligned}
$$

By observing that the $\left[H_{0}\right\rfloor^{n} u_{k} \neq 0$ due to the fact that $\left[\begin{array}{lll}\left(\left\lceil H_{0}\right\rfloor^{n}\right)^{T} & \cdots & \Omega^{(N-1) / 2}\left(\left\lceil H_{0}\right\rfloor^{n}\right)^{T}\end{array}\right]^{T}=V_{N} \bar{H}$ is of full-column rank, the proof is completed.

PROOF OF THEOREM 4: By noting that the feasibility of (20) ensures (44), the assertion readily follows from the above proof for Theorem 3 .

\section{ACKNOWLEDGMENT}

The authors would like to thank the anonymous reviewers, and the guest editors, G. Chesi and D. Henrion, for their careful reading and valuable suggestions, and $\mathrm{M}$. Araki, President of Matsue National College of Technology, Japan, for his enthusiastic guidance when he taught at Kyoto University.

\section{REFERENCES}

[1] V. Balakrishnan and L. Vandenberghe, "Semidefinite programming duality and linear time-invariant systems," IEEE Trans. Automat. Control, vol. 48, no. 1, pp. 30-41, Jan. 2003

[2] B. R. Barmish, New Tools for Robustness of Linear Systems. London, U.K.: Macmillan, 1994.

[3] D. P. Bertsekas, Nonlinear Programming. Belmont, MA: Athena Scientific, 1999.

[4] S. P. Boyd, L. E. Ghaoui, E. Feron, and V. Balakrishnan, Linear Matrix Inequalities in System and Control Theory. Philadelphia, U.K.: SIAM, 1998.

[5] P. A. Bliman, "A convex approach to robust stability for linear systems with uncertain scalar parameters," SIAM J. Control Optim., vol. 42, no. 6, pp. 2016-2042, 2004.

[6] P. A. Bliman, "An existence result for polynomial solutions of parameter-dependent LMIs,” Syst. Control Lett., vol. 51, pp. 165-169, 2004.

[7] G. Chen and T. Sugie, "New upper bound of the real $\mu$ based on the parameter dependent multiplier," in Proc. Conf. Decision Control, 1996, pp. 1293-1294. 
[8] G. Chesi, A. Garulli, A. Tesi, and A. Vicino, "Polynomially parameter-dependent Lyapunov functions for robust stability of polytopic systems: An LMI approach," IEEE Trans. Automat. Control, vol. 50, no. 3, pp. 365-370, Mar. 2005.

[9] Y. Ebihara and T. Hagiwara, "A dilated LMI approach to robust performance analysis of linear time-invariant uncertain systems," Automatica, vol. 41, no. 11, pp. 1933-1941, 2005.

[10] Y. Ebihara and T. Hagiwara, "On the degree of polynomial parameterdependent Lyapunov functions for robust stability of single parameterdependent LTI systems: A counter-example to Barmish's conjecture," Automatica, vol. 42, no. 9, pp. 1599-1603, 2006.

[11] L. E. Ghaoui, F. Oustry, and H. Lebret, "Robust solutions to uncertain semidefinite programs," SIAM J. Optim., vol. 9, no. 1, pp. 33-52, 1998.

[12] T. Hagiwara, "Inertia approach to the Hamiltonian eigenvalue tests for frequency-domain inequality conditions," Dynam. Continuous, Discrete Impulsive Syst., Series B: Appl. Algorithms, pp. 279-283, 2005.

[13] D. Henrion and G. Meinsma, "Rank-one LMIs and Lyapunov's inequality," IEEE Trans. Automat. Control, vol. 46, no. 8, pp. 1285-1288, Aug. 2001.

[14] D. Henrion, D. Arzelier, D. Peaucelle, and J. B. Lasserre, "On parameter-dependent Lyapunov functions for robust stability of linear systems," in Proc. Conf. Decision Control, 2004, pp. 887-892.

[15] D. Henrion and J. B. Lasserre, "Detecting global optimality and extracting solutions in gloptipoly," in Lecture Notes in Control and Information Sciences. New York: Springer Verlag, 2005, vol. 312.

[16] D. Henrion and J. B. Lasserre, "Convergent relaxations of polynomial matrix inequalities and static output feedback," IEEE Trans. Automat. Control, vol. 51, no. 2, pp. 192-202, Feb. 2006.

[17] R. A. Horn and C. A. Johnson, Matrix Analysis. Cambridge, U.K.: Cambridge University Press, 1995.

[18] T. Iwasaki and S. Hara, "Well-posedness of feedback systems: Insight into exact robustness analysis and approximate computations," IEEE Trans. Automat. Control, vol. 43, no. 5, pp. 619-630, May 1998.

[19] T. Iwasaki, G. Meinsma, and M. Fu, "Generalized $\mathcal{S}$-procedure and finite frequency KYP Lemma," Math. Problems Eng., vol. 6, pp. 305-320, 2000

[20] T. Iwasaki and G. Shibata, "LPV system analysis via quadratic separator for uncertain implicit systems," IEEE Trans. Automat. Control, vol. 46, no. 8, pp. 1195-1208, Aug. 2001.

[21] T. I. Iwasaki and S. Hara, "Generalized KYP Lemma: Unified frequency domain inequalities with design applications," IEEE Trans. Automat. Control, vol. 50, no. 1, pp. 41-59, Jan. 2005.

[22] J. B. Lassere, "Global optimization with polynomials and the problem of moments," SIAM J. Optim., vol. 11, no. 3, pp. 796-817, 2001.

[23] G. Meinsma, Y. Shrivastava, and M. Fu, "A dual formulation of mixed $\mu$ and on the losslessness of $(D, G)$ scaling," IEEE Trans. Automat. Control, vol. 42, no. 7, pp. 1032-1036, Jul. 1997.

[24] R. C. L. F. Oliveira and P. L. D. Peres, "Stability of polytopes of matrices via affine parameter-dependent Lyapunov functions," Linear Algebra Appl., vol. 405, no. 1, pp. 209-228, 2005.

[25] A. Packard and J. C. Doyle, "The complex structured singular value," Automatica, vol. 29, no. 1, pp. 71-109, 1993.

[26] P. A. Parrilo, "Semidefinite programming relaxations for semialgebraic problems," Math. Programm., vol. 96, pp. 293-320, 2003.

[27] A. Rantzer, "On the Kalman-Yakubovich-Popov Lemma," Syst. Control Lett., vol. 28, pp. 7-10, 1996

[28] C. W. Scherer, "LPV control and full block multipliers," Automatica, vol. 37 , no. 3, pp. 361-375, 2001.

[29] C. W. Scherer, "LMI relaxations in robust control: How to reduce conservatism?," in Proc. Plenary Talk IFAC Symp. Robust Control Design, 2003, [CD ROM]
[30] C. W. Scherer, "Relaxations for robust linear matrix inequality problems with verifications for exactness," SIAM J. Matrix Anal. Appl., vol. 27, no. 2, pp. 365-395, 2005.

[31] C. W. Scherer, "LMI relaxations in robust control," Eur. J. Control, vol. 12, no. 1, pp. 3-29, 2006

[32] C. W. Scherer and C. W. J. Hol, "Matrix sum-of-squares relaxations for robust semi-definite programs," Math. Programm., vol. 107, pp. $189-211,2006$

[33] J. F. Sturm, "Using SeDuMi 1.02, a MATLAB toolbox for optimization over symmetric cones," Optim. Methods Software, vol. 11-12, pp. 625-653, 1999.

[34] X. Zhang, P. Tsiotras, and T. Iwasaki, "Parameter-dependent Lyapunov functions for exact stability analysis of single-parameter dependent LTI systems," in Proc. Conf. Decision Control, 2003, pp. 5168-5173.

[35] K. Zhou and J. C. Doyle, Essentials of Robust Control. Englewood Cliffs, NJ: Prentice Hall, 1998.

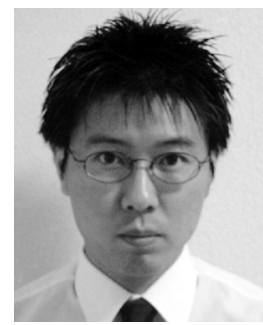

Yoshio Ebihara (M'03) was born in Fukuoka, Japan, on May 12, 1974. He received the B.E., M.E., and D.E. degrees in electrical engineering from Kyoto University, Kyoto, Japan, in 1997, 1999 and 2002, respectively.

Since 2002, he has been with the Department of Electrical Engineering, Kyoto University, where he has been a Lecturer since 2006. His research interests include computer-aided control system analysis and design.

Dr. Yoshio Ebihara was the Student Best Paper Finalist at the American Control Conference, Anchorage, AK, in 2002.

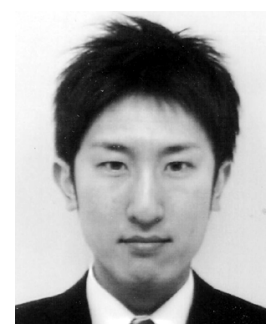

Yusuke Onishi was born in Hyogo, Japan, on June 14, 1983. He received the B.E. degree in electronic engineering from Osaka University, Osaka, Japan, in 2006, and the M.E. degree in electrical engineering from Kyoto University, Kyoto, Japan, in 2008.

Since 2008, he has been with Kansai Telecasting Corporation, Osaka.

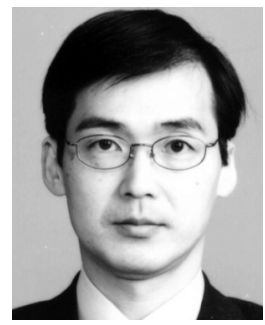

Tomomichi Hagiwara (M'96) was born in Osaka, Japan, on March 28, 1962. He received the B.E., M.E., and D.E. degrees in electrical engineering from Kyoto University, Kyoto, Japan, in 1984, 1986 and 1990, respectively.

Since 1986, he has been with the Department of Electrical Engineering, Kyoto University, where he has been a Professor since 2001. His research interests include dynamical system theory and control theory such as analysis and design of sampled-data systems, time-delay systems, and two-degree-of-freedom control systems. 